NEIP-00-005

hep-th/0003078

March 2000

\title{
On the Effective $\mathrm{N}=1$ Supergravity of M-Theory ${ }^{\star}$
}

\author{
J.-P. Derendinger and R. Sauser ${ }^{\dagger}$ \\ Institute of Physics, University of Neuchâtel \\ CH-2000 Neuchâtel, Switzerland
}

\begin{abstract}
In the low-energy limit, M-theory compactified on $S^{1} / \mathbf{Z}_{2}$ is formulated in terms of Bianchi identities with sources localized at orbifold singularities and anomaly-cancelling counterterms to the Wilson effective Lagrangian. Compactifying to four dimensions on a Calabi-Yau space leads to $N=1$ local supersymmetry. We derive a formulation of the effective supergravity which explicitly relates four-dimensional supergravity multiplets and field equations with these fundamental M-theory aspects. This formulation proves convenient for the introduction in the effective supergravity of nonperturbative M-theory contributions. It also applies to the universal sector of generic compactifications with $N=1$ supersymmetry.
\end{abstract}

* Research supported in part by the Swiss National Science Foundation.

$\dagger$ jean-pierre.derendinger, roger.sauser@iph.unine.ch 


\section{Introduction}

M-theory compactified on

$$
O_{7}=X_{6} \times S^{1} / \mathbf{Z}_{2}
$$

where $X_{6}$ is a Calabi-Yau three-fold, leads to a four-dimensional theory with $N=1$ local supersymmetry. At present, our knowledge of the resulting effective supergravity is based on the few aspects of M-theory which are quantitatively understood and on the small-orbifold limit which is the perturbative heterotic $E_{8} \times E_{8}$ string compactified on the Calabi-Yau space $X_{6}$. In the low-energy limit, M-theory information can be organized as an expansion in powers of the eleven-dimensional gravitational constant $\kappa_{11}$ [1, 2]. The lowest order $\kappa_{11}^{-2}$ is eleven-dimensional supergravity [3]. In the case of a compactification on $S^{1} / \mathbf{Z}_{2}$ only, the next orders in $\kappa_{11}$ are known to include orbifold plane contributions (super-Yang-Mills terms) as well as gauge and gravitational anomaly-cancelling terms [1, 4, 2].

Similarly, the effective four-dimensional supergravity[ can be formulated as an expansion in the four-dimensional gravitational constant $\kappa$, even if a more common choice suggested by string theory is to use the dilaton as expansion parameter. The two options coincide provided the appropriate supersymmetric description of the dilaton is adopted in a Wilson effective Lagrangian formulation. The lowest order $\kappa^{-2}$ is the $O_{7}$ truncation of eleven-dimensional supergravity. The next order includes super-YangMills, charged matter kinetic and superpotential contributions. Then come sigmamodel anomaly-cancelling terms contributing in particular to gauge threshold corrections. These first corrections to the low-energy limit of compactified M-theory are identical to those obtained from heterotic compactifications on Calabi-Yau. This is certainly expected since the information content is identical. The literature gives a detailed description of these results, with particular attention paid to the "strongcoupling' heterotic limit in which the size of the Calabi-Yau space is smaller than the orbifold length, supersymmetry breaking by gaugino condensation and non-standard embeddings [5]- 8].

Our goal in this paper is to provide a derivation of the effective supergravity which explicitly relates four-dimensional supergravity statements with M-theory aspects like

\footnotetext{
${ }^{1}$ The limit in which the length of the $S^{1} / \mathbf{Z}_{2}$ orbifold direction is small compared to the size of the Calabi-Yau manifold.

${ }^{2}$ Which is a low-energy description.
} 
Bianchi identities modified at singularities and anomaly cancellation. We reformulate these basic facts of M-theory on $O_{7}$ directly in terms of four-dimensional supermultiplets and equations. For instance, Bianchi identities from M-theory are promoted to field equations, as constraints on multiplets which are massless modes of M-theory bulk fields. With this formulation, we expect to obtain a clean, direct derivation of the effective supergravity suitable to cases more subtle than the universal modes of M-theory on $\mathrm{O}_{7}$. A first use of our formalism will be the coupling of five-brane moduli supermultiplets in the $\mathrm{O}_{7}$ case [9].

The organization of the paper is as follows. In Section \&, we establish our basic supergravity formulation, using the bosonic bulk dynamics as starting point. The resulting Lagrangian is the lowest order in the $\kappa$-expansion. It is essentially defined by a dynamical Lagrangian involving tensor fields supplemented by Bianchi identities which are field equations of the theory. We discuss in detail the bosonic component expansion of the supermultiplet action, the question of the gravity frame and the generation of a superpotential. In Section 3, we introduce the next order corrections: gauge multiplets and charged matter contributions. We show that their introduction is controlled by a simple modification of the four-dimensional Bianchi identities, in analogy with the appearance of $\mathbf{Z}_{2}$ fixed planes contributions in the M-theory Bianchi identities. Section

4 discusses anomaly-cancelling terms. We begin by modifying the four-dimensional effective supergravity by adding terms similar to those appearing for gauge threshold corrections in $(2,2)$ compactifications of the heterotic string. These modifications can be formulated in terms of our particular set of multiplets directly related to M-theory bulk degrees of freedom. We then directly compute these anomaly-cancelling terms by Kaluza-Klein reduction of the ten-dimensional Green-Schwarz counterterms arising from M-theory on $S^{1} / \mathbf{Z}_{2}$. Section 5 gives some final comments and conclusions, and an appendix contains our notations and conventions.

\section{Four-dimensional effective M-theory supergravities: bulk dynamics}

Our concern is compactifications of M-theory to four dimensions preserving $N=1$ supersymmetry. Or compactifications in which supersymmetry would break spontaneously or dynamically at solutions of the effective field equations. As a consequence, 
the light (massless) modes can be described by a local effective $N=1$ supergravity Lagrangian, to be understood in the sense of Wilson.

These solutions are not as well understood as, for instance, Calabi-Yau compactifications of heterotic strings and it will prove useful to give a precise description of the aspects which are better known. We will then focus on a precise description of two aspects which are of importance in compactifications of M-theory. Firstly, we will use a supersymmetric description of the Bianchi identities verified by antisymmetric tensors, as arising from M-theory or higher-dimensional supergravities. This procedure allows to avoid multiplet ambiguities arising when duality transformations are performed at the bosonic level only. Secondly, we will use a formulation which leaves explicitly the choice of gravity frame open. This can be a relevant issue since an expansion, perturbative or not, is performed around a gravitational background which selects a gravity frame. Standard Poincaré supergravity is usually written in the Einstein frame, in which the gravitational Lagrangian is $-\frac{1}{2 \kappa^{2}} e R$. Corrections to the lowest order effective action, which includes this gravitational term, induce in general (but not always) corrections to the gravitational Lagrangian which affect the Einstein frame condition.

Keeping open the choice of gravity frame suggests to use superconformal supergravity. And displaying Bianchi identities explicitly in an effective Lagrangian requires using chiral, linear or vector supermultiplets with constraints.

The purpose of this first section is to establish our procedure by considering the wellknown 'bulk dynamics', which follows from $O_{7}$ compactification of eleven-dimensional supergravity.

\subsection{Superconformal formalism}

We use the superconformal formulation of $N=1$ supergravity with a chiral compensating multiplet $S_{0}$ to generate Poincaré theories by gauge fixing' Its conformal and chiral weights are taken as $w=1$ and $n=1$. This formalism is particularly convenient to keep control of a change of frameft which corresponds to a different Poincaré gauge condition applied on the modulus of the scalar compensator $z_{0}$, which fixes dilatation

\footnotetext{
${ }^{3}$ This is 'old minimal' Poincaré supergravity 10 .

${ }^{4}$ Mostly the so-called Einstein or string frames.
} 
symmetry. Up to two derivatives, a supergravity Lagrangian is written as

$$
\mathcal{L}=\left[S_{0} \bar{S}_{0} \Phi\right]_{D}+\left[S_{0}^{3} W\right]_{F}+\frac{1}{4}\left[f_{a b} \mathcal{W}^{a} \mathcal{W}^{b}\right]_{F}
$$

The symbols $[\ldots]_{D}$ and $[\ldots]_{F}$ denote respectively the invariant $D$ - and $F$-density formulæ given by (all fermion contributions are omitted)

$$
\begin{aligned}
& {[\mathcal{V}]_{D}=e\left(d+\frac{1}{3} c R\right),} \\
& {[\mathcal{S}]_{F}=e(f+\bar{f}),}
\end{aligned}
$$

where $\mathcal{V}$ is a vector multiplet with components $\left(c, \chi, m, n, b_{\mu}, \lambda, d\right)$ and $\mathcal{S}$ a chiral multiplet with components $(z, \psi, f)$. The real vector multiplet $\Phi$ with zero weights is a function (in the sense of tensor calculus) of the multiplets present in the theory, including in general the compensating multiplet. The holomorphic function $W$ of the chiral multiplets is the superpotential. The last term contributes to gauge kinetic terms (the chiral multiplet $\mathcal{W}$ is the gauge field strength for the gauge multiplets) and involves a holomorphic gauge kinetic function $f_{a b}$ of the chiral multiplets ${ }^{\emptyset}$. Expression (2.1) provides the most general supergravity Lagrangian up to terms with more than two derivatives and up to terms which would contribute to kinetic terms in a fermionic background only $\left[12\right.$, 13. Besides $S_{0}$ and $\mathcal{W}$, we will use chiral multiplets with zero weights and neither $W$ nor $f_{a b}$ will depend on the compensator.

The chiral $U(1)$ symmetry of the superconformal algebra can be extended to

$$
S_{0}, \quad W, \quad \Phi \quad \longrightarrow \quad \Lambda S_{0}, \quad \Lambda^{-3} W,\left.\quad(\Lambda \bar{\Lambda})^{-1} \Phi\right|_{S_{0} \rightarrow \Lambda S_{0}}
$$

with an arbitrary chiral multiplet $\Lambda$. This symmetry is at the origin of Kähler invariance of Poincaré supergravity. The last transformation suggests that $\log \Phi$ transforms as the corresponding gauge connection. Choosing $\Lambda=W^{1 / 3}$ eliminates the superpotential except if it vanishes. One can then use a $U(1) /$ Kähler gauge fixing in which the supergravity Lagrangian (2.1) reads

$$
\mathcal{L}=\left[S_{0} \bar{S}_{0} \Phi\right]_{D}+c\left[S_{0}^{3}\right]_{F}+\frac{1}{4}\left[f_{a b} \mathcal{W}^{a} \mathcal{W}^{b}\right]_{F}
$$

with an arbitrary constant $c$ as superpotential and two arbitrary functions $\Phi$ and $f_{a b}$.

\footnotetext{
${ }^{5}$ Except otherwise mentioned, our notation is as in ref. [11] where also reference to the original literature can be found.

${ }^{6}$ Gauge kinetic terms may also arise from the first term.
} 
The real function $\Phi$ depends on matter multiplets, which will be either chiral multiplets like the Calabi-Yau universal modulus $T$, or real linear multiplets (with weights $w=2, n=0$ ) like the dilaton multiplet in the version of the theory with an antisymmetric tensor, or real vector multiplets $(n=0, w$ arbitrary) like the multiplet of gauge potentials $(w=0)$. Vector multiplets will appear as essential ingredients in the effective description of $(N=1)$-preserving M-theory five-branes [9].

\subsection{Bulk Lagrangian}

The lowest order (in the $\kappa$ expansion) effective four-dimensional supergravity of Mtheory compactified on $\mathrm{O}_{7}$ describes Kaluza-Klein massless modes of eleven-dimensional supergravity. It is the $S^{1} / \mathbf{Z}_{2}$ truncation of eleven-dimensional supergravity on a Calabi-Yau three-fold.

In the version given by Cremmer, Julia and Scherk (CJS) [3], the Lagrangian of eleven-dimensional supergravity can be written as]

$$
\begin{aligned}
e^{-1} \mathcal{L}_{\mathrm{CJS}}= & \frac{1}{2 \kappa_{11}^{2}}\left[-R-\frac{1}{2 \cdot 4 !} G_{M_{1} M_{2} M_{3} M_{4}} G^{M_{1} M_{2} M_{3} M_{4}}\right. \\
& \left.-\frac{1}{6} \frac{1}{4 ! 4 ! 3 !} e^{-1} \epsilon^{M_{1} \ldots M_{11}} G_{M_{1} M_{2} M_{3} M_{4}} G_{M_{5} M_{6} M_{7} M_{8}} C_{M_{9} M_{10} M_{11}}\right] \\
& + \text { fermionic terms. }
\end{aligned}
$$

Omitting all fields related to the detailed geometry of the Calabi-Yau manifold, the particle content of the four-dimensional theory is the $N=1$ supergravity multiplet, with metric tensor $g_{\mu \nu}$, and matter multiplets including (on-shell) four bosons and four fermions. Two bosons are scalars and correspond to the dilaton and the "universal modulus' of the Calabi-Yau space, the massless volume mode. Two bosons are KaluzaKlein modes of the four-form field $G$, with Bianchi identity $d G=0$. Explicitly, these fields and their Bianchi identities can be written as

$$
\begin{array}{ll}
G_{\mu \nu \rho 4}, & \partial_{[\mu} G_{\nu \rho \sigma 4]}=0, \\
G_{\mu j \bar{k} 4}=i T_{\mu} \delta_{j \bar{k}}, & \partial_{[\mu} T_{\nu]}=0 .
\end{array}
$$

It will prove useful to identify these fields with the vector components of two real vector multiplets $V$ and $V_{T}$, and to impose the Bianchi identities as field equations using the

\footnotetext{
${ }^{7}$ We use the flat space-time metric $(-,+,+, \ldots,+)$. The gravitational constant $\kappa_{11}$ has dimension (mass $)^{-9 / 2}$ and the three-index tensor field $C_{M N P}$ is dimensionless. Notations and conventions are defined in an appendix.
} 
appropriate multiplets as Lagrange multipliers. The bulk supergravity Lagrangian takes then the form

$$
\mathcal{L}_{\mathrm{B}}=\left[-\frac{1}{\sqrt{2}}\left(S_{0} \bar{S}_{0} V_{T}\right)^{3 / 2} V^{-1 / 2}-(S+\bar{S}) V+L_{T} V_{T}\right]_{D} .
$$

The vector superfield $V$ (weights $w=2, n=0$ ) includes in its components the vector field $v^{\mu} \propto \epsilon^{\mu \nu \rho \sigma} G_{\nu \rho \sigma 4}$ for which the Bianchi identity is $\partial_{\mu} v^{\mu}=0$. This condition is a component of the (super)field equation of the chiral $S(w=n=0)$ which imposes $V=L$, a real linear multiplet $(w=2, n=0)$. Secondly, the vector multiplet $V_{T}$ $(w=n=0)$ includes in its components $T_{\mu}$. The supersymmetric extension of its Bianchi identity $\partial_{[\mu} T_{\nu]}=0$ is enforced by the real linear multiplet $L_{T}$, which implies $V_{T}=T+\bar{T}$, with a chiral weightless multiplet $T$. The usefulness of obtaining Bianchi identities via field equations will become apparent with the introduction of higher orders in the $\kappa$ expansion. At this stage of the discussion however, it gives a formulation of the familiar duality relating scalars and antisymmetric tensors or, for superfields, chiral and linear multiplets.

Solving in Eq. (2.7) for the Lagrange multipliers $S$ and $L_{T}$ leads to the "standard form' of the bulk four-dimensional Lagrangian [14, 15]

$$
\mathcal{L}_{\mathrm{B}, \mathrm{l}}=-\frac{1}{\sqrt{2}}\left[\left(S_{0} \bar{S}_{0} e^{-\hat{K} / 3}\right)^{3 / 2} L^{-1 / 2}\right]_{D},
$$

or, as defined in Eq. (2.1),

$$
\Phi=-\left(\frac{2 L}{S_{0} \bar{S}_{0}}\right)^{-1 / 2} e^{-\hat{K} / 2}
$$

The Kähler potential for the volume modulus $T$ is

$$
\hat{K}=-3 \log (T+\bar{T})
$$

We will see again below that this standard form is naturally obtained by direct reduction of the CJS version of eleven-dimensional supergravity on $O_{7}$. Clearly, theory (2.8) is also the Calabi-Yau truncation of ten-dimensional $N=1$ pure supergravity [14. Notice that $\hat{K}$ can be regarded as the Kähler connection for symmetry (2.3), with transformation

$$
\hat{K} \quad \longrightarrow \quad \hat{K}+3 \log \Lambda+3 \log \bar{\Lambda},
$$

such that $S_{0} \bar{S}_{0} e^{-\hat{K} / 3}$ is chiral/Kähler invariant. 
A theory with a linear multiplet is in principle dual to an equivalent Lagrangian with the linear multiplet replaced by a chiral one. In our case, solving for $V$ and $L_{T}$ in expression (2.7) leads to

$$
\begin{aligned}
\mathcal{L}_{\mathrm{B}, \mathrm{c}} & =-\frac{3}{2}\left[S_{0} \bar{S}_{0} e^{-K / 3}\right]_{D}, \\
K & =-\log (S+\bar{S})+\hat{K}=-\log (S+\bar{S})-3 \log (T+\bar{T}) .
\end{aligned}
$$

This familiar chiral form [16] is not the most useful as long as one insists on the fourdimensional translation of eleven-dimensional Bianchi identities.

Notice that one can obtain another equivalent form of the Lagrangian (2.7) by choosing to solve for $S$ and $V_{T}$. In this case, the Calabi-Yau modulus is described by a linear multiplet $L_{T}$. This form will not be useful since it is known that one-loop string corrections in general break the chiral-linear duality for this modulus: they involve holomorphic functions of $T$ in a $F$-density which are intrinsically chiral [17]. Finally, there is an obstruction when trying to solve for $V$ and $V_{T}$ and one cannot write an expression in terms of the chiral $S$ and the linear $L_{T}$.

Before turning to explicit component expressions, we should discuss the choice of Poincaré frame, and introduce the expansion in the four-dimensional gravity coupling $\kappa$, which effectively corresponds to the low-energy expansion of M-theory in powers of the eleven-dimensional gravitational constant $\kappa_{11}$.

\subsubsection{Einstein frame}

To gauge-fix dilatations, we impose as usual a condition on the Einstein term appearing in the superconformal supergravity Lagrangian. According to the component expression for the $D$-density and the tensor calculus of superconformal multiplets [11], the Einstein term included in $\left[S_{0} \bar{S}_{0} \Phi\right]_{D}$ is 10 , 15

$$
\mathcal{L}_{\mathrm{E}}=-\frac{1}{2} e R\left[-\frac{2}{3} z_{0} \bar{z}_{0}\left(\Phi-\frac{1}{2} \sum_{i} w_{i} C_{i} \frac{\partial \Phi}{\partial C_{i}}\right)\right]
$$

where $w_{i}$ is a Weyl weight, the sum is taken over the linear $\left(w_{i}=2\right)$ and vector $\left(w_{i}\right.$ arbitrary) multiplets, and $z_{0}, \Phi$ and $C_{i}$ are the lowest, scalar components of respectively $S_{0}, \Phi$ and of the vector or linear multiplets?

\footnotetext{
${ }^{8}$ We use in general the same notation for the lowest component of $\Phi$ and the multiplet itself. Also, in expression (2.13), we use the component expansion of vector multiplets indicated below [Eq. [2.23)], which differs in its highest component from ref. [11].
} 
Applied to the bulk Lagrangian (2.7), expression (2.13) leads to

$$
\mathcal{L}_{\mathrm{E}}=-\frac{1}{2} e R\left[\left(z_{0} \bar{z}_{0} C_{T}\right)^{3 / 2}(2 C)^{-1 / 2}\right] \text {. }
$$

As they should, the terms introduced to impose Bianchi identities do not contribute. The Einstein frame is then selected by the dilatation gauge condition

$$
\kappa^{-2}=\left(z_{0} \bar{z}_{0} C_{T}\right)^{3 / 2}(2 C)^{-1 / 2}
$$

It will be convenient to introduce the (composite) real vector multiplet

$$
\Upsilon=\left(S_{0} \bar{S}_{0} V_{T}\right)^{3 / 2}(2 V)^{-1 / 2}
$$

with conformal weight two. In the Poincaré theory and in the Einstein frame, its lowest component is precisely equal to $\kappa^{-2}$. With this definition, the bulk Lagrangian becomes simply

$$
\mathcal{L}_{\mathrm{B}}=\left[-\Upsilon-(S+\bar{S}) V+L_{T} V_{T}\right]_{D},
$$

and the equation of motion for $V$ (the chiral-linear duality equation)

$$
2 V(S+\bar{S})=\Upsilon
$$

indicates that the Einstein Lagrangian also reads

$$
\mathcal{L}_{\mathrm{E}}=-(2 C \operatorname{Re} s) e R
$$

In the Einstein frame (Planck units), Re $s=\left(4 \kappa^{2} C\right)^{-1}$.

Eq. (2.18) is compatible with the standard relation of heterotic strings $2 \kappa^{2}\langle\operatorname{Re} s\rangle=$ $\alpha^{\prime}$ if one identifies $2\langle C\rangle=1 / \alpha^{\prime}$. This equation defines string units, in which

$$
\mathcal{L}_{\mathrm{E}}=-\frac{e^{-2 \varphi}}{\alpha^{\prime}} e R
$$

with a dilaton given by $e^{-2 \varphi}=\operatorname{Re} s$.

\subsubsection{Modified Bianchi identities and $\kappa$-expansion}

Compactification of M-theory on $S^{1} / \mathbf{Z}_{2}$ is commonly discussed in an expansion in powers of $\kappa_{11}$. Compactification on $O_{7}$ can similarly be formulated with $\kappa$ as expansion parameter. In the upstairs version, Bianchi identities are modified at the tendimensional planes fixed by $S^{1} / \mathbf{Z}_{2}$. Suppose now that we modify the four-dimensional

\footnotetext{
${ }^{9}$ We use $\langle\ldots\rangle$ for a background value.
} 
supersymmetric Bianchi identities of the bulk Lagrangian in the following way:

$$
\mathcal{L}_{\mathrm{B}} \quad \longrightarrow \quad \mathcal{L}=\left[-\Upsilon-(S+\bar{S})\left(V+\Delta_{V}\right)+L_{T}\left(V_{T}+\Delta_{T}\right)\right]_{D},
$$

with two composite vector multiplets $\Delta_{V}(w=2, n=0)$ and $\Delta_{T}(w=n=0)$. Solving for the Lagrange multipliers now leads to

$$
V=L-\Delta_{V}, \quad V_{T}=T+\bar{T}-\Delta_{T}
$$

The Lagrangian to first order in these modifications reads then

$$
\begin{aligned}
\mathcal{L} & =\mathcal{L}_{\mathrm{B}}-\left[\frac{\Upsilon}{2 V} \Delta_{V}-\frac{3}{2} \frac{\Upsilon}{V_{T}} \Delta_{T}\right]_{D} \\
& =\mathcal{L}_{\mathrm{B}}-\left[(S+\bar{S}) \Delta_{V}-\frac{3}{2 V_{T}}\left(\Upsilon \Delta_{T}\right)\right]_{D},
\end{aligned}
$$

with $V$ and $V_{T}$ respectively replaced by $L$ and $T+\bar{T}$ in these expressions. The multiplets $\Delta_{V}$ and $\Upsilon \Delta_{T}$, with 'canonical' dimension $w=2$, appear at order $\Upsilon^{0} \sim \kappa^{0}$, in comparison with bulk terms of order $\Upsilon \sim \kappa^{-2}$. This is the relation with the expansion in powers of $\kappa_{11}$ of M-theory in the low-energy limit. In M-theory compactification, the multiplets $\Delta_{V}$ and $\Delta_{T}$ can thus be obtained either by considering the modified Bianchi identities on $\mathrm{O}_{7}$, formulated as in Eq. (2.20), or from corrections to the Lagrangian of eleven-dimensional supergravity on $O_{7}$, as in expression (2.21).

\subsubsection{Component expressions}

To analyze the Lagrangian (2.7), we will need to define some notations. Since we will only explicitly consider the bosonic sector of the theory, all fermions in the $N=1 \mathrm{su}$ permultiplets will be omitted. Since also we are concerned with Poincaré supergravity, we will immediately gauge-fix the superconformal symmetries not contained in $N=1$ Poincaré supersymmetry, with one exception, dilatation symmetry: we want to keep the freedom of a frame choice as explicit as possible. These assumptions imply in particular that superconformal covariant derivatives reduce in general to $D_{\mu}^{c} \phi=D_{\mu} \phi-\frac{i}{2} n A_{\mu} \phi$ for a complex field with chiral weight $n$ and to

$$
\square^{c} \varphi=\square \varphi+\frac{1}{6} w \varphi R
$$

if $w$ is the Weyl weight and $\varphi$ real. The gauge boson $A_{\mu}$ of chiral $U(1)$ symmetry is auxiliary and $D_{\mu}$ and $\square$ would be covariantized with respect to Poincaré symmetries. 
We will use the following components for the various superconformal multiplets appearing in Lagrangian (2.7):

$$
\begin{aligned}
V & =\left(C, 0, H, K, v_{\mu}, 0, d-\square C-\frac{1}{3} C R\right), \\
V_{T} & =\left(C_{T}, 0, H_{T}, K_{T}, T_{\mu}, 0, d_{T}-\square C_{T}\right), \\
S & =\left(s, 0,-f, i f, i \partial_{\mu} s, 0,0\right), \\
L_{T} & =\left(\ell_{T}, 0,0,0, t_{\mu}, 0,-\square \ell_{T}-\frac{1}{3} \ell_{T} R\right), \quad t_{\mu}=\frac{e}{2} \epsilon_{\mu \nu \rho \sigma} \partial^{\nu} t^{\rho \sigma}, \\
S_{0} & =\left(z_{0}, 0,-f_{0}, i f_{0}, i D_{\mu}^{c} z_{0}, 0,0\right) .
\end{aligned}
$$

The role of the Lagrange multipliers $S$ and $L_{T}$ follows from

$$
\begin{aligned}
e^{-1}[(S+\bar{S}) V]_{D}= & 2\left(\partial^{\mu} \operatorname{Im} s\right) v_{\mu}-2 \partial^{\mu}\left(\operatorname{Re} s \partial_{\mu} C\right)+2 d \operatorname{Re} s \\
& -f(H-i K)-\bar{f}(H+i K) \\
= & -2 \operatorname{Im} s \partial^{\mu} v_{\mu}+2 d \operatorname{Re} s-f(H-i K)-\bar{f}(H+i K) \\
& + \text { derivative, } \\
e^{-1}\left[L_{T} V_{T}\right]_{D}= & \ell_{T}\left(d_{T}-\square C_{T}\right)-\frac{e}{2} \epsilon_{\mu \nu \rho \sigma}\left(\partial^{\mu} T^{\nu}\right) t^{\rho \sigma}+\text { derivative. }
\end{aligned}
$$

Solving for the components of $S$ leads to $\partial^{\mu} v_{\mu}=d=H=K=0$, and $V$ is a linear multiplet. Solving for the components of $L_{T}$ leads to $d_{T}-\square C_{T}=\partial_{[\mu} T_{\nu]}=0$, and $V_{T}$ can be written as $T+\bar{T}$, with a chiral multiplet $T$ (zero weights) $\square$.

Since one can always write $v_{\mu}=\frac{e}{6} \epsilon_{\mu \nu \rho \sigma} v^{\nu \rho \sigma}$, we have generated with $\operatorname{Im} s$ and $t_{\mu \nu}$ the Bianchi identities

$$
\partial_{[\mu} v_{\nu \rho \sigma]}=\partial_{[\mu} T_{\nu]}=0
$$

A modification of these Bianchi identities, as induced by $S^{1} / \mathbf{Z}_{2}$ compactification or by five-brane couplings will then be phrased as a modification of the supermultiplets appearing multiplied by $S+\bar{S}$ or $L_{T}$ in Eqs. (2.24).

To complete the identification of the four-dimensional supergravity (2.7) with the modes of eleven-dimensional supergravity we need its complete bosonic expansion, which after solving for the chiral $U(1)$ auxiliary field $A_{\mu}$ reads:

$$
\begin{aligned}
e^{-1} \mathcal{L}_{\mathrm{B}}= & -\frac{1}{2} \Upsilon R+\frac{1}{4} \Upsilon C^{-2} v_{\mu} v^{\mu}-\frac{3}{4} \Upsilon C_{T}^{-2} T_{\mu} T^{\mu}-t_{\mu} T^{\mu}+2 \operatorname{Im} s \partial_{\mu} v^{\mu} \\
& -\frac{1}{4} \Upsilon C^{-2}\left(\partial_{\mu} C\right)\left(\partial^{\mu} C\right)-\frac{3}{4} \Upsilon C_{T}^{-2}\left(\partial_{\mu} C_{T}\right)\left(\partial^{\mu} C_{T}\right) \\
& +d\left(\frac{1}{2} \Upsilon C^{-1}-2 \operatorname{Re} s\right)+\left(d_{T}-\square C_{T}\right)\left(\ell_{T}-\frac{3}{2} \Upsilon C_{T}^{-1}\right) \\
& +\frac{1}{2}\left(\partial_{\mu} \Upsilon\right)\left[\partial^{\mu} \log C+\partial^{\mu} \log \Upsilon\right] \\
& +e^{-1} \mathcal{L}_{\text {AUX. }}+\text { derivative }
\end{aligned}
$$

\footnotetext{
10 The components are: $C_{T}=2 \operatorname{Re} T, T_{\mu}=-2 \partial_{\mu} \operatorname{Im} T, H_{T}=-2 \operatorname{Re} f_{T}, K_{T}=-2 \operatorname{Im} f_{T}$.
} 
where $\Upsilon=\left(z_{0} \bar{z}_{0} C_{T}\right)^{3 / 2}(2 C)^{-1 / 2}$, and

$$
\begin{aligned}
e^{-1} \mathcal{L}_{\mathrm{AUX} .}= & -\frac{1}{4} \Upsilon C^{-2}(H+i K)(H-i K)+\frac{3}{4} \Upsilon C_{T}^{-2}\left(H_{T}+i K_{T}\right)\left(H_{T}-i K_{T}\right) \\
& +f(H-i K)+\bar{f}(H+i K) .
\end{aligned}
$$

The last equality is obtained after solving for the $f_{0}$ component of $S_{0} \square$.

The above component expansion of the bosonic Lagrangian is useful because it explicitly displays the dependence on the gauge choice for dilatation symmetry. The gravitational constant is the field-dependent quantity $\Upsilon$. Choosing a Poincaré frame amounts to impose the value of this quantity, and to use this condition to eliminate $z_{0}$. Notice also that the choice of the phase of $z_{0}$ is a gauge condition for the chiral internal $U(1)$ superconformal symmetry. With the exception of $z_{0}$, all bosons are $U(1)$-neutral. As a consequence, the bosonic Lagrangian only depends on the modulus of $z_{0}$.

Taking the Einstein frame, $\Upsilon=\kappa^{-2}$, and solving for the components of $S$ and $L_{T}$ leads to

$$
\begin{aligned}
e^{-1} \mathcal{L}_{\mathrm{B}}= & -\frac{1}{2 \kappa^{2}} R-\frac{1}{4 \kappa^{2}} C^{-2}\left[\left(\partial_{\mu} C\right)\left(\partial^{\mu} C\right)-v_{\mu} v^{\mu}\right] \\
& -\frac{3}{4 \kappa^{2}} C_{T}^{-2}\left[\left(\partial_{\mu} C_{T}\right)\left(\partial^{\mu} C_{T}\right)+T_{\mu} T^{\mu}\right]
\end{aligned}
$$

with $v_{\mu}=\frac{e}{2} \epsilon_{\mu \nu \rho \sigma} \partial^{\nu} b^{\rho \sigma}$ since $V$ is a linear multiplet, $C_{T}=2 \operatorname{Re} T$ and $T_{\mu}=-2 \partial_{\mu} \operatorname{Im} T$ since $V_{T}=T+\bar{T}$. This Lagrangian is to be compared with the reduction of the CJS version of eleven-dimensional supergravity (2.5). The $\mathbf{Z}_{2}$ orbifold projection eliminates all states which are odd under $x^{4} \rightarrow-x^{4}$, and since we disregard massless modes related to the detailed Calabi-Yau geometry, the reduction of the $D=11$ space-time metric is

$$
g_{M N}=\left(\begin{array}{ccc}
e^{-\gamma} e^{-2 \sigma} g_{\mu \nu} & 0 & 0 \\
0 & e^{2 \gamma} e^{-2 \sigma} & 0 \\
0 & 0 & e^{\sigma} \delta_{i \bar{j}}
\end{array}\right) .
$$

The $S U(3)$-invariant tensor $\delta_{i \bar{j}}$ refers to complex coordinates on the Calabi-Yau space. The surviving components of the four-index tensor $G_{M N P Q}$ are only $G_{\mu \nu \rho 4}$ and $G_{\mu i \bar{j} 4}$, with

$$
G_{\mu \nu \rho 4}=3 \partial_{[\mu} C_{\nu \rho] 4}, \quad G_{\mu i \bar{j} 4}=\partial_{\mu} C_{i \bar{j} 4}, \quad C_{i \bar{j} 4}=i a(x) \delta_{i \bar{j}},
$$

and the four-dimensional Lagrangian for these fields reads

$$
\begin{aligned}
e^{-1} \mathcal{L}_{\mathrm{CJS}}= & -\frac{1}{2 \kappa^{2}} R-\frac{1}{4 \kappa^{2}}\left[9\left(\partial_{\mu} \sigma\right)\left(\partial^{\mu} \sigma\right)+\frac{1}{6} e^{6 \sigma} G_{\mu \nu \rho 4} G^{\mu \nu \rho 4}\right] \\
& -\frac{3}{4 \kappa^{2}}\left[\left(\partial_{\mu} \gamma\right)\left(\partial^{\mu} \gamma\right)+e^{-2 \gamma}\left(\partial_{\mu} a\right)\left(\partial^{\mu} a\right)\right]
\end{aligned}
$$

\footnotetext{
${ }^{11}$ Note that the result would be different with a superpotential.
} 
In this expression, $\kappa$ is the four-dimensional gravitational coupling

$$
\kappa^{2}=\frac{\kappa_{11}^{2}}{V_{7}}
$$

$V_{7}=V_{1} V_{6}$ being the volume of the compact space $S^{1} \times X_{6}$.

At this stage, the identification of the bosonic components $C$ and $b_{\mu \nu}$ of $V, C_{T}$ and $T_{\mu}$ of $V_{T}$ with the bulk fields $\sigma, \gamma, C_{\mu \nu 4}$ and $a$ can only be determined up to a proportionality constant for each multiplet. We will define these constants later on from the couplings of $C$ and $C_{T}=2 \operatorname{Re} T$ to charged matter and gauge fields, to obtain:

$$
\begin{array}{ll}
4 \kappa^{2} C=\frac{\lambda^{2}}{V_{6}} e^{-3 \sigma}, & 4 \kappa^{2} b_{\mu \nu}=\frac{\lambda^{2}}{V_{6}} C_{\mu \nu 4}, \\
C_{T}=2 \frac{\lambda^{2}}{V_{6}} e^{\gamma}=2 \operatorname{Re} T, & T_{\mu}=-2 \frac{\lambda^{2}}{V_{6}} \partial_{\mu} a=-2 \partial_{\mu} \operatorname{Im} T .
\end{array}
$$

The quantity $\lambda$ is the gauge coupling constant on the $\mathbf{Z}_{2}$ fixed planes and $\lambda^{2} / V_{6}$ is a dimensionless number which will actually never appear in the four-dimensional effective theory.

\subsubsection{Bianchi identities and symmetries}

In the bulk Lagrangian (2.7), the terms $\left[-(S+\bar{S}) V+L_{T} V_{T}\right]_{D}$ impose in particular the Bianchi identities (2.6). They are certainly invariant under

$$
\begin{aligned}
& V \quad \longrightarrow \quad V+L, \quad L \text { linear, } \\
& V_{T} \quad \longrightarrow \quad V_{T}+T+\bar{T}, \quad T \text { chiral. }
\end{aligned}
$$

These symmetries are the supersymmetric extensions of the gauge invariances of Bianchi identities, $\delta G_{\mu \nu \rho 4}=3 \partial_{[\mu} \Lambda_{\nu \rho]}$ and $\delta G_{\mu i \bar{j} 4}=i \partial_{\mu} \Lambda \delta_{i \bar{j}}$. Solving for $S$ and $L_{T}$ implies then that $V$ and $V_{T}$ are 'pure gauge', $V=L$ and $V_{T}=T+\bar{T}$. The last equation defines $V_{T}$ up to a holomorphic redefinition of $T, T \rightarrow f(T)$. This redefinition is a symmetry of the bulk Lagrangian if the function $\Phi$ simultaneously transforms as in (2.3), with $\Lambda$ a holomorphic function of $T$. The equation for the invariance of $S_{0} \bar{S}_{0} V_{T}$ is

$$
f(T)+\bar{f}(\bar{T})=\frac{T+\bar{T}}{\Lambda(T) \bar{\Lambda}(\bar{T})},
$$

and its solution is clearly $S l(2, \mathbf{R})$ symmetry,

$$
T \quad \longrightarrow \quad f(T)=\frac{a T-i b}{i c T+d}, \quad a d-b c=1,
$$

the modular invariance of $T$ (T-duality), extended to a continuous symmetry at the lowest order. 
This chiral symmetry is generically anomalous: in the presence of a $N=1$ superYang-Mills sector, with or without chiral matter, mixed anomalies arise in the triangle diagram for two gauge bosons and one connection $-3 \log (T+\bar{T})$ for $S l(2, \mathbf{R})$ symmetry. This anomaly is cancelled in particular by a Green-Schwarz mechanism as was demonstrated in the effective Lagrangian description of gauge thresholds 13 calculated at one-loop for $(2,2)$ compactifications of the heterotic strings [17. We will see below that this phenomenon is also a useful tool in the construction of effective supergravities of M-theory compactifications.

\subsubsection{Superpotential}

The standard reduction of eleven-dimensional supergravity with unbroken $N=1$ supersymmetry does not generate a superpotential. This fact is however not a direct consequence of the eleven-dimensional Bianchi identity or of the Calabi-Yau and $S^{1} / \mathbf{Z}_{2}$ symmetries. In principle, the Bianchi identity $\partial_{[M} G_{N P Q R]}=0$ allows a solution

$$
G_{i j k 4}=2 i \kappa^{-1} h \epsilon_{i j k}, \quad G_{\overline{i j k} 4}=-2 i \kappa^{-1} h \epsilon_{i j k} .
$$

In these equations, $h$ is a constant chosen real and $\epsilon_{i j k}$ is the $S U(3)$-invariant CalabiYau tensor. The Lagrangian term $-\frac{e}{2 \kappa_{11}^{2}} \frac{1}{48} G_{M N P Q} G^{M N P Q}$ leads then to a contribution

$$
-\frac{e}{\kappa^{4}} C_{T}^{-3}\left(2 \kappa^{2} C\right) h^{2}
$$

in the four-dimensional effective supergravity. This contribution corresponds to the addition of a superpotential term

$$
\left[i h S_{0}^{3}\right]_{F}
$$

to the bulk Lagrangian, a contribution which however breaks supersymmetry 18. Since we have insisted in writing Lagrangians in which all Bianchi identities are field equations, we prefer instead to use

$$
[U(W+\bar{W})]_{D}+\left[S_{0}^{3} W\right]_{F}
$$

The field equation of the vector multiplet $U$ (weights $w=2, n=0$ ) implies that the chiral multiplet $W(w=n=0)$ is an arbitrary imaginary constant, which can be zero and supersymmetry stays unbroken, or non-zero. 
With the addition (2.33) of a superpotential, the bulk Lagrangian takes its final 'off-shell' form

$$
\mathcal{L}_{\mathrm{B}}=\left[-\Upsilon-(S+\bar{S}) V+L_{T} V_{T}+U(W+\bar{W})\right]_{D}+\left[S_{0}^{3} W\right]_{F},
$$

in which the Bianchi identities of eleven-dimensional supergravity are translated into field equations of the Lagrange multipliers $S, L_{T}$ and $U$. At this stage, the introduction of these multiplets is not fascinating. This approach encodes simply the (Poincaré) dualities relating antisymmetric tensors (in linear multiplets) and scalars (in chiral multiplets), and the Bianchi identity for the superpotential is trivial. But this procedure will prove useful and informative in the forthcoming sections.

\section{Fixed planes: gauge and matter contributions}

In this section, we start with the well-known effective $N=1$ four-dimensional supergravity for symmetric $(2,2)$ compactifications of heterotic strings. We then rewrite this theory in a form where explicit Bianchi identities allow a direct comparison with $\mathrm{O}_{7}$ compactification of M-theory, in the so-called upstairs formulation [1, 2].

The dependence on charged matter (in chiral multiplets collectively denoted by $M$, with $w=n=0$ ) and gauge multiplets (vector multiplet $A$, in the adjoint representation, with $w=n=0$ ) of the effective supergravity theory for Calabi-Yau compactifications of heterotic strings is well-known [16, 19, 20], at least for the 'universal' matter multiplets arising from the simplest Calabi-Yau modes of the ten-dimensional superYang-Mills fields. Information on the non-trivial harmonic modes is more subtle [21], as for generic Calabi-Yau moduli. In the chiral formulation, Eq. (2.12) becomes

$$
\begin{aligned}
\mathcal{L}_{\mathrm{c}} & =-\frac{3}{2}\left[S_{0} \bar{S}_{0} e^{-K / 3}\right]_{D}+\left[\frac{1}{4} S \mathcal{W W}+S_{0}^{3} W\right]_{F}, \\
K & =-\log (S+\bar{S})-3 \log \left(T+\bar{T}-2 \bar{M} e^{A} M\right) \\
W & =\alpha M^{3}
\end{aligned}
$$

For notational simplicity, we omit traces over the gauge group representation and their normalisation factors. The chiral multiplet $\mathcal{W}$ is the gauge field-strength for $A$ $(w=n=3 / 2)$. Since the gauge group is in general not simple,

$$
\mathcal{W} \mathcal{W}=\sum_{a} c^{a} \mathcal{W}^{a} \mathcal{W}^{a}
$$


with a (real) coefficient $c^{a}$ for each simple or abelian factor ${ }^{\mathrm{T}}$. The superpotential should be understood as a gauge invariant trilinear interaction with coupling constant $\alpha$ defined as an integral over the Calabi-Yau space. In the linear multiplet version, the equivalent expression is [14, 15]

$$
\mathcal{L}_{1}=-\frac{1}{\sqrt{2}}\left[\left(S_{0} \bar{S}_{0}\right)^{3 / 2} \hat{L}^{-1 / 2} e^{-\hat{K} / 2}\right]_{D}+\left[\alpha S_{0}^{3} M^{3}\right]_{F}
$$

With respect to Eq. (2.8), gauge and matter dependence arises in modifications of the linear multiplet $L$ (to $\hat{L}$ ) and of $\hat{K}$ : the new modulus and matter Kähler potential is

$$
\hat{K}=-3 \log \left(T+\bar{T}-2 \bar{M} e^{A} M\right)
$$

instead of Eq. (2.10) and

$$
\hat{L}=L-2 \Omega
$$

where $\Omega(A)$ is the Chern-Simons vector multiplet $(w=2, n=0)$, defined by ${ }^{13}$

$$
\Omega=\sum_{a} c^{a} \Omega^{a}, \quad \Sigma\left(\Omega^{a}\right)=\frac{1}{16} \mathcal{W}^{a} \mathcal{W}^{a}
$$

Insisting as before on Bianchi identities, both forms (3.1) and (3.3) are equivalent to

$$
\begin{aligned}
\mathcal{L}= & {\left[-\left(S_{0} \bar{S}_{0} V_{T}\right)^{3 / 2}(2 V)^{-1 / 2}-(S+\bar{S})(V+2 \Omega)\right.} \\
& \left.+L_{T}\left(V_{T}+2 \bar{M} e^{A} M\right)+\left\{U\left(W-\alpha M^{3}\right)+\text { c.c. }\right\}\right]_{D}+\left[S_{0}^{3} W\right]_{F} \\
= & {\left[-\Upsilon-(S+\bar{S})(V+2 \Omega)+L_{T}\left(V_{T}+2 \bar{M} e^{A} M\right)\right]_{D}+\left[S_{0}^{3}\left(i h+\alpha M^{3}\right)\right]_{F} . }
\end{aligned}
$$

Supersymmetric vacua have $h=0$. As before, solving in the last expression for $S$ and $L_{T}$ imposes respectively $V=L-2 \Omega=\hat{L}$ and $V_{T}=T+\bar{T}-2 \bar{M} e^{A} M$, leading to Eq. (3.3). Alternatively, solving for $V$ and $L_{T}$ leads back to the chiral form (3.1), with the tensor calculus identity

$$
-2[(S+\bar{S}) \Omega]_{D}=\frac{1}{4} \sum_{a} c^{a}\left[S \mathcal{W}^{a} \mathcal{W}^{a}\right]_{F}+\text { derivative }
$$

which follows from Eq. (3.6) and the definition of the $F$-density, $[\Sigma(\ldots)]_{F}=-[\ldots]_{D}$.

\footnotetext{
${ }^{12}$ Corresponding to Kac-Moody levels in superstrings. All coefficients can be equal to one, as with the 'standard embedding', but our discussion is not affected by their presence.

13 In global Poincaré supersymmetry, $\Sigma(\Omega)=-\frac{1}{4} \overline{D D} \Omega$. A linear multiplet is defined by the condition $\Sigma(L)=0$.
} 
This reformulation of the gauge invariant Lagrangian suggests some remarks. Firstly, it enhances the importance of Chern-Simons multiplets in superstring effective actions: gauge fields and matter fields couple to the bulk Lagrangian using a ChernSimons multiplet. The gauge Chern-Simons multiplet $\Omega$ is defined by Eq. (3.6), which indicates that its chiral projection $\Sigma(\Omega)$ is the chiral multiplet for the kinetic super-Yang-Mills Lagrangian. Similarly for chiral matter, the kinetic Wess-Zumino Lagrangian can be written as $\left[S_{0} \bar{S}_{0} \bar{M} e^{A} M\right]_{D}=-\left[\Sigma\left(S_{0} \bar{S}_{0} \bar{M} e^{A} M\right)\right]_{F}$, defining

$$
\Omega_{M}=S_{0} \bar{S}_{0} \bar{M} e^{A} M
$$

as a matter Chern-Simons multiplet $(w=2, n=0)$ which then couples to $S_{0} \bar{S}_{0} V_{T}$ as $\Omega$ couples to $V$.

Secondly, the Chern-Simons vector multiplet $\Omega(A)$ is not gauge invariant: its variation is a linear multiplet. Then, the variation of $[(S+\bar{S}) \Omega]_{D}$ is a derivative and $V$ remains gauge invariant. When solving for $S$, it simply follows that $\hat{L}$ is gauge invariant and that the linear multiplet transforms as

$$
\delta L=2 \delta \Omega
$$

Finally, expression (3.7) shows that all gauge and chiral matter contributions can be viewed as the supersymmetrization of modified Bianchi identities imposed by $S, L_{T}$ and $U$. This is equally true in the ten-dimensional supergravity-Yang-Mills system: the curl of the antisymmetric tensor field is modified by Chern-Simons contributions which are supersymmetry partners of the super-Yang-Mills Lagrangian [22]. This observation provides the link to the approach based on M-theory on $O_{7}$, in which the $\mathbf{Z}_{2}$-fixed planes carrying the Yang-Mills fields induce because of supersymmetry modifications to the Bianchi identity of the four-form field strength of eleven-dimensional supergravity.

In the effective supergravity of M-theory on $O_{7}$ ('upstairs formulation'), the various components of the Lagrangian (3.7),

$$
\begin{aligned}
\mathcal{L}= & {\left[-\left(S_{0} \bar{S}_{0} V_{T}\right)^{3 / 2}(2 V)^{-1 / 2}-(S+\bar{S})(V+2 \Omega)\right.} \\
& \left.+L_{T}\left(V_{T}+2 \bar{M} e^{A} M\right)+\left\{U\left(W-\alpha M^{3}\right)+\text { c.c. }\right\}\right]_{D}+\left[S_{0}^{3} W\right]_{F},
\end{aligned}
$$

have the following origin. As already discussed at length, the first term is the bulk supergravity contribution. Then $[(S+\bar{S})(V+2 \Omega)]_{D}$ is the supersymmetrization of the 
Bianchi identity verified by the component $G_{\mu \nu \rho 4}$ of the four-form field, modified by gauge contributions on the fixed planes. Similarly, $\left[L_{T}\left(V_{T}+2 \bar{M} e^{A} M\right)\right]_{D}$ and $[U(W-$ $\left.\alpha M^{3}\right)+$ c.c. $]_{D}$ are respectively the supersymmetric extensions of the Bianchi identities of $G_{\mu j \bar{k} 4}$ and $G_{i j k 4}$, when fixed plane contributions are included. Thus, all fixed plane contributions are given at this order by the supersymmetrization of Bianchi identities, as obtained by direct $O_{7}$ truncation of the eleven-dimensional identities [1] [ ]

At this point, the gauge coupling constant for each simple or abelian factor $a$ in the gauge group appears to be

$$
\frac{1}{g_{a}^{2}}=c^{a} \operatorname{Re} s=\frac{c^{a} \Upsilon}{4 C}
$$

$s$ and $C$ being respectively the lowest scalar component of the chiral $S$ and the vector $V$ (or the linear $L$ ). At this order, $g_{a}$ is the tree-level wilsonnian and physical gauge coupling. The second equality is the lowest component of the equation of motion of the vector multiplet $V$, Eq. (2.18). In the Einstein frame, $\operatorname{Re} s=\left(4 \kappa^{2} C\right)^{-1}$.

It is clear, as already observed [5]-[7], that as far as the structure of the fourdimensional effective supergravity is concerned, the same information follows from $O_{7}$ compactification of M-theory at the next to lowest order in the $\kappa$ expansion and from Calabi-Yau compactifications of the heterotic strings, at zero string loop order.

Notice that Eq. (3.11) defines $c^{a} \operatorname{Re} s$ as the coefficient of gauge kinetic terms. It defines then this field in terms of the gauge kinetic action on the ten-dimensional $\mathbf{Z}_{2}$ fixed planes,

$$
\mathcal{S}_{\text {gauge }}=-\frac{1}{4 \lambda^{2}} \int_{M_{10}} d^{10} x e_{10} \operatorname{tr} F_{A B} F^{A B},
$$

reduced on $X_{6}$. This action is also at the origin of charged matter kinetic terms, which in the effective supergravity read

$$
-\frac{e}{\kappa^{2}} \frac{\partial^{2} \hat{K}}{\partial \bar{M} \partial M}\left(D_{\mu} \bar{M}\right)\left(D^{\mu} M\right)=-\frac{6 e}{\kappa^{2} C_{T}}\left(D_{\mu} \bar{M}\right)\left(D^{\mu} M\right)+\ldots
$$

The Calabi-Yau reduction of the action (3.12) provides then the identification of $C$ and $C_{T}$ in terms of the bulk fields $\sigma$ and $\gamma$ appearing in the metric tensor (2.28). These results have already been displayed in Eq. (2.30).

\footnotetext{
${ }^{14}$ The coefficient of $-\frac{1}{4} F_{\mu \nu}^{a} F^{a \mu \nu}$ in the generating functional of one-particle irreducible Green's functions.
} 


\section{Anomaly-cancelling terms}

In the ten-dimensional heterotic string, cancellation of gauge and gravitational anomalies is a one-loop effect in string or effective supergravity perturbation theory. In the low-energy effective action description, we should then distinguish the Wilson effective supergravity from the standard effective action $\mathcal{S}_{\Gamma}$, defined as the generating functional of one-particle irreducible Green's functions. The latter action can be obtained in a diagrammatic expansion built from the Wilson Lagrangian $\mathcal{L}$, itself obtained from string perturbation theory as an expansion

$$
\mathcal{L}=\mathcal{L}^{(0)}+\mathcal{L}^{(1)}+\ldots
$$

the subscript being the string-loop order. The expressions given in the previous sections were for $\mathcal{L}^{(0)}$, or for the tree-level $\mathcal{S}_{\Gamma}$. At the string one-loop level, $\mathcal{S}_{\Gamma}$ includes tree and one-loop diagrams generated by the Feynman rules of the tree-level Wilson Lagrangian $\mathcal{L}^{(0)}$. These include anomalous loop diagrams. It also includes tree diagrams generated by the Green-Schwarz counterterm [23] introduced in $\mathcal{L}^{(1)}$ to cancel the anomalies generated by $\mathcal{L}^{(0)}$. The mechanism for symmetry restoration implies that $\mathcal{L}^{(1)}$ is not invariant under the restored symmetry.

In four space-time dimensions, the nature of the cancelled anomalies is known from studies of $(2,2)$ compactifications of heterotic strings in the Yang-Mills sector [17, 13, 24]: target-space duality of the modulus $T$ has a one-loop anomaly which is cancelled by a counterterm in $\mathcal{L}^{(1)}$, in a generalization to sigma-model anomalies of the Green-Schwarz mechanism [25]. The derivation of the complete counterterm requires a calculation to all orders in the modulus $T$ [17]. However, at the present stage of understanding, the M-theory approach should be regarded as a large- $T$ limit in which T-duality reduces to a shift symmetry in the imaginary part of $T$.

Our goal in this section is to obtain some or all counterterms in $\mathcal{L}^{(1)}$ associated with anomaly-cancellation in the low-energy description. We are particularly interested in contributions to gauge kinetic terms, the so-called threshold corrections. And we want to formulate these terms using the 'M-theory multiplets' $V, V_{T}$ and $W$ corresponding to the surviving components of $G$, in contrast to the 'heterotic multiplets' $S$ (or $L$ ) and $T$. We begin by obtaining the relevant information from the case of heterotic $(2,2)$ symmetric orbifolds. 


\subsection{Information from symmetric $(2,2)$ orbifolds}

Retaining only the universal modulus $T$ and a linear dilaton multiplet $L$, the Wilson one-loop Lagrangian for heterotic symmetric $(2,2)$ orbifolds includes a term

$$
\mathcal{L}^{(1)}=-2 \delta_{G S}[\hat{L} \log (T+\bar{T})]_{D}+\sum_{a} b^{a}\left[\log \eta(i T) \mathcal{W}^{a} \mathcal{W}^{a}\right]_{F},
$$

where $\delta_{G S}$ and $b^{a}$ are numbers depending on the orbifold and $\eta(i T)$ is the Dedekind function. Under $\operatorname{Sl}(2, \mathbf{Z})$ T-duality 2.31), the variation of $\mathcal{L}^{(1)}$ is

$$
\begin{aligned}
\delta \mathcal{L}^{(1)} & =2 \delta_{G S}[\hat{L}\{\log \varphi(T)+\log \bar{\varphi}(\bar{T})\}]_{D}+\frac{1}{2} b^{a}\left[\log \varphi(T) \mathcal{W}^{a} \mathcal{W}^{a}\right]_{F} \\
& =\frac{1}{2}\left(\delta_{G S}+b^{a}\right)\left[\log \varphi(T) \mathcal{W}^{a} \mathcal{W}^{a}\right]_{F},
\end{aligned}
$$

with $\varphi(T)=i c T+d$. On the other hand, the triangle one-loop diagram for two gauge fields and one Kähler connection $-3 \log (T+\bar{T})$ is anomalous. Its variation is

$$
\delta \Delta=\frac{1}{2} A^{a}\left[\log \varphi(T) \mathcal{W}^{a} \mathcal{W}^{a}\right]_{F},
$$

where $A^{a}$ is the chiral-anomaly coefficient, as obtained from the expression of the diagram. The anomaly cancels since one finds that $b^{a}+\delta_{G S}+A^{a}=0$ for all factors in the gauge group (the index $a$ ). The one-loop correction to gauge kinetic terms obtained from the component expansion of $\mathcal{L}^{(1)}$ and from the triangle diagram reads

$$
-\frac{1}{4} F_{\mu \nu}^{a} F^{a \mu \nu}\left[-\left(\delta_{G S}+A^{a}\right) \log (T+\bar{T})+b^{a} \log |\eta(i T)|^{4}\right] .
$$

It is modular invariant since the anomaly is cancelled, and its value is controlled by the $F$-density contribution to $\mathcal{L}^{(1)}$, with coefficients $b^{a}$.

The Wilson Lagrangian depends on the coefficients $\delta_{G S}$ and $b^{a}$. But the information on gauge thresholds is in the numbers $b^{a}$. In general, the parameters of the Wilson Lagrangian computed at a non-trivial loop order are not of direct physical significance and this is here the case of $\delta_{G S}$ in the sector of gauge kinetic terms.

In the large- $T$ limit, T-duality reduces to $\operatorname{Im} T \rightarrow \operatorname{Im} T+$ constant, the Kähler connection $-3 \log (T+\bar{T})$ is invariant and, strictly speaking, no anomaly survives to be cancelled. In addition, $\log |\eta(i T)|^{4} \sim-\frac{\pi}{3}(T+\bar{T})$ dominates the logarithmic contributions. The threshold correction is then of the simple form

$$
-\frac{1}{4} \sum_{a}\left[-\frac{\pi b^{a}}{3}(T+\bar{T})\right] F_{\mu \nu}^{a} F^{a \mu \nu},
$$

\footnotetext{
${ }^{15}$ In this paragraph, we use $c^{a}=1$ and $\hat{L}=L-2 \sum_{a} \Omega^{a}$.
} 
invariant under the imaginary shift symmetry of $T$. Its supersymmetrization is

$$
-\frac{\pi}{12} \sum_{a} b^{a}\left[T \mathcal{W}^{a} \mathcal{W}^{a}\right]_{F}=\frac{2 \pi}{3} \sum_{a} b^{a}\left[(T+\bar{T}) \Omega^{a}\right]_{D} .
$$

As long as $T$ only is considered, there seems to be no way to identify the $D$-density contribution to $\mathcal{L}^{(1)}$ in the large- $T$ limit. The introduction of the matter multiplet $M$ changes the picture. In the lowest order Wilson Lagrangian $\mathcal{L}^{(0)}$, the Kähler connection $-3 \log (T+\bar{T})$ is modified to $-3 \log \left(T+\bar{T}-2 \bar{M} e^{A} M\right)$. As a consequence, the contribution with coefficient $A^{a}$ to the gauge threshold (4.2) involves the quantity $\log (T+\bar{T}-2 \bar{M} M)$. Then, either the one-loop term $\mathcal{L}^{(1)}$ is accordingly modified to $-2 \delta_{G S}\left[\hat{L} \log \left(T+\bar{T}-2 \bar{M} e^{A} M\right)\right]_{D}$ and the parameter $\delta_{G S}$ disappears from gauge thresholds, or, less plausibly, this is not the case and $\delta_{G S}$ acquires a physical significance in $M$-dependent thresholds. In any case, since the holomorphic Dedekind function cannot depend on $\bar{M} e^{A} M$, a calculation of the correction $\mathcal{L}^{(1)}$ to the Wilson Lagrangian to first order in $\bar{M} e^{A} M$ will give access to the $D$-density Green-Schwarz term and to the parameter $\delta_{G S}$.

From the point of view of M-theory on $\mathrm{O}_{7}$ or heterotic strings on Calabi-Yau, $\bar{M} e^{A} M$ arises from the ten-dimensional Chern-Simons terms, at the same order as gauge kinetic terms. This indicates that the $D$-density contribution to $\mathcal{L}^{(1)}$ should be visible in a reduction of the ten-dimensional Green-Schwarz terms.

\subsection{The case of M-theory on $O_{7}$}

Before embarking in a derivation of the anomaly-cancelling terms from the low-energy limit of M-theory on $\mathrm{O}_{7}$, we consider the problem at the level of four-dimensional supergravity only.

In the large- $T$ limit, as discussed in the previous paragraph, the $T$-dependent corrections to gauge kinetic terms are of the form

$$
\frac{1}{4} \sum_{a} \beta^{a}\left[T \mathcal{W}^{a} \mathcal{W}^{a}\right]_{F}
$$

with coefficients which are in principle calculable in heterotic strings. To rewrite them in terms of the 'M-theory multiplets', we first note that

$$
\frac{1}{4} \sum_{a} \beta^{a}\left[T \mathcal{W}^{a} \mathcal{W}^{a}\right]_{F}=-2\left[(T+\bar{T}) \sum_{a} \beta^{a} \Omega^{a}\right]_{D}
$$


Since the field equation of the Lagrange multiplier $L_{T}$ implies that $V_{T}=T+\bar{T}-$ $2 \bar{M} e^{A} M$, this expression can also be written

$$
-2\left[\left(V_{T}+2 \bar{M} e^{A} M\right) \sum_{a} \beta^{a} \Omega^{a}\right]_{D} .
$$

The right-hand side of Eq. (4.4) is gauge invariant because $\delta \Omega^{a}$ is a linear multiplet and therefore $\left[(T+\bar{T}) \delta \Omega^{a}\right]_{D}$ is a derivative. To ensure gauge invariance of expression (4.5), we add the term $\left[L_{T}\left(V_{T}+2 \bar{M} e^{A} M\right)\right]_{D}$ included in Lagrangian (3.7). We obtain

$$
\left[\left(L_{T}-2 \sum_{a} \beta^{a} \Omega^{a}\right)\left(V_{T}+2 \bar{M} e^{A} M\right)\right]_{D},
$$

which is gauge invariant if we postulate that

$$
\delta L_{T}=2 \sum_{a} \beta^{a} \delta \Omega^{a} .
$$

The correction (4.4) to the super-Yang-Mills Lagrangian is independent of the matter fields and has a holomorphic character (it can be seen as a correction to the holomorphic gauge kinetic function $f_{a b}$ ). To enumerate possible matter-dependent contributions to gauge kinetic terms, we consider for simplicity a single matter multiplet $M$ transforming in some unspecified representation of the gauge group. The first candidate counterterm is a real density:

$$
\sum_{a} \gamma^{a}\left[\bar{M} e^{A} M \Omega^{a}\right]_{D}
$$

Gauge invariance requires however its appearance in the combination

$$
-2 \delta\left[\bar{M} e^{A} M\left(L-2 \sum_{a} c^{a} \Omega^{a}\right)\right]_{D}+2 \gamma\left[\bar{M} e^{A} M\left(L_{T}-2 \sum_{a} \beta^{a} \Omega^{a}\right)\right]_{D}
$$

or

$$
-2 \delta\left[\bar{M} e^{A} M V\right]_{D}+2 \gamma\left[\bar{M} e^{A} M\left(L_{T}-2 \sum_{a} \beta^{a} \Omega^{a}\right)\right]_{D},
$$

using 'M-theory multiplet' $V$. In the first counterterm, each gauge group factor contributes with weight $c^{a}$, as in lowest order terms. The second contribution can be combined with expression (4.6) into

$$
\left[\left(L_{T}-2 \sum_{a} \beta^{a} \Omega^{a}\right)\left(V_{T}+2(1+\gamma) \bar{M} e^{A} M\right)\right]_{D} .
$$

We will also see below that the M-theory anomaly-cancelling terms generate a contribution of the form

$$
\epsilon\left[V\left|\alpha M^{3}\right|^{2}\right]_{D}
$$


involving the matter superpotential. Since the factor $1+\gamma$ in expression (4.8) can be eliminated by a rescaling of $M$, of $\delta$ and of the superpotential coupling constant $\alpha$, we may take $\gamma=0$ at our level of approximation, and the Wilson Lagrangian up to string one-loop order is expected to become

$$
\begin{aligned}
\mathcal{L}= & {\left[-\Upsilon-(S+\bar{S})(V+2 \Omega)+\left\{U\left(W-\alpha M^{3}\right)+\text { c.c. }\right\}\right.} \\
& +\left(L_{T}-2 \sum_{a} \beta^{a} \Omega^{a}\right)\left(V_{T}+2 \bar{M} e^{A} M\right) \\
& \left.+V\left(\epsilon\left|\alpha M^{3}\right|^{2}-2 \delta \bar{M} e^{A} M\right)\right]_{D}+\left[S_{0}^{3} W\right]_{F} .
\end{aligned}
$$

Notice that the contributions which correspond to string one-loop effects do not include any correction to the Einstein Lagrangian, which remains simply

$$
-\frac{1}{2} \Upsilon e R, \quad \Upsilon=\left(z_{0} \bar{z}_{0} C_{T}\right)^{\frac{3}{2}}(2 C)^{-\frac{1}{2}},
$$

and the Einstein frame condition remains $\Upsilon=\kappa^{-2}$. This is expected since the gravitational constant in the heterotic string is not corrected at string one-loop order.

From the general expression (4.10) in which Bianchi identities are field equations for $S, L_{T}$ and $U$, we can derive various equivalent forms. For instance, solving for $S, L_{T}$ and $U$ leads to the version of the effective supergravity in which the dilaton is described by a linear multiplet:

$$
\begin{aligned}
\mathcal{L}_{\mathrm{l}}= & {\left[-\left(S_{0} \bar{S}_{0}\right)^{3 / 2}\left(T+\bar{T}-2 \bar{M} e^{A} M\right)^{3 / 2}(2 \hat{L})^{-1 / 2}\right]_{D}+\left[S_{0}^{3}\left(i h+\alpha M^{3}\right)\right]_{F} } \\
& +\left[\hat{L}\left(\epsilon\left|\alpha M^{3}\right|^{2}-2 \delta \bar{M} e^{A} M\right)\right]_{D}+\left[\frac{1}{4} T \sum_{a} \beta^{a} \mathcal{W}^{a} \mathcal{W}^{a}\right]_{F} .
\end{aligned}
$$

The second line is a one-loop correction in the perturbative expansion of the heterotic string in which $\hat{L}$ is the string loop-counting field [14]. Its $T$-dependent part corresponds to the Green-Schwarz counterterm found in [13] for symmetric heterotic orbifolds. Each of these one-loop corrections, with coefficients $\epsilon, \delta$ and $\beta^{a}$, is related to a well-defined counterterm which can be easily identified in, for instance, the low-energy limit of Mtheory on $O_{7}$. The Green-Schwarz counterterms controlled by $\delta$ and $\epsilon$ are intrinsically real $D$-densities. They will appear as corrections to the Kähler potential, as matterdependent 'wave-function renormalisations', in the dual version with a chiral dilaton multiplet. On the other hand, the holomorphic $T$-dependent terms are true threshold corrections.

Alternatively, solving for $L_{T}, V$ and $U$ leads to the version with a chiral dilaton 
multiplet:

$$
\mathcal{L}_{\mathrm{c}}=-\frac{3}{2}\left[S_{0} \bar{S}_{0} e^{-K / 3}\right]_{D}+\frac{1}{4}\left[\sum_{a}\left(c^{a} S+\beta^{a} T\right) \mathcal{W}^{a} \mathcal{W}^{a}\right]_{F}+\left[S_{0}^{3}\left(i h+\alpha M^{3}\right)\right]_{F},
$$

with the Kähler potential"

$$
\begin{aligned}
K= & -\log \left(S+\bar{S}+2 \delta \bar{M} e^{A} M-\epsilon\left|\alpha M^{3}\right|^{2}\right) \\
& -3 \log \left(T+\bar{T}-2 \bar{M} e^{A} M\right)
\end{aligned}
$$

and the gauge kinetic functions $f^{a}=c^{a} S+\beta^{a} T$. An ambiguity exists however because one can perform a holomorphic redefinition of the two chiral multiplets. For instance,

$$
S=\tilde{S}-\delta T
$$

leads to the equivalent Kähler potential

$$
\begin{aligned}
K= & -\log \left(\tilde{S}+\overline{\tilde{S}}-\delta\left(T+\bar{T}-2 \bar{M} e^{A} M\right)-\epsilon\left|\alpha M^{3}\right|^{2}\right) \\
& -3 \log \left(T+\bar{T}-2 \bar{M} e^{A} M\right)
\end{aligned}
$$

with gauge kinetic functions $f^{a}=c^{a} \tilde{S}+\left(\beta^{a}-c^{a} \delta\right) T$. The origin of this ambiguity at the level of 'M-theory multiplets' is interesting. Suppose that we add the counterterm

$$
\Delta \mathcal{L}=A\left[(V+2 \Omega)\left(V_{T}+2 \bar{M} e^{A} M\right)\right]_{D}
$$

to the fundamental Lagrangian (4.10), with an arbitrary constant $A$. The theory becomes then

$$
\begin{aligned}
\mathcal{L}_{A}= & {\left[-\Upsilon-(S+\bar{S})(V+2 \Omega)+\left\{U\left(W-\alpha M^{3}\right)+\text { c.c. }\right\}\right.} \\
& +\left(L_{T}-2 \sum_{a}\left(\beta^{a}-A c^{a}\right) \Omega^{a}\right)\left(V_{T}+2 \bar{M} e^{A} M\right) \\
& \left.+V\left(\epsilon\left|\alpha M^{3}\right|^{2}+A V_{T}+2(A-\delta) \bar{M} e^{A} M\right)\right]_{D}+\left[S_{0}^{3} W\right]_{F} .
\end{aligned}
$$

It is gauge invariant provided the appropriate transformation of $L_{T}$ is postulated. We have apparently obtained a family of four-dimensional supergravities, depending on a new parameter $A$. This is however only true before solving for the Lagrange multiplier multiplets. Firstly, solving for $S$ and $L_{T}$ leads to the space-time derivative $\Delta \mathcal{L}=A[L(T+\bar{T})]_{D}$. The counterterm $\Delta \mathcal{L}$ is then irrelevant in the version of the

\footnotetext{
${ }^{16}$ The superfield Kähler potential includes covariantization contributions $e^{A}$ which disappear in the bosonic expression used in component expansions.
} 
theory with a linear dilaton. Secondly, if we instead solve for $V$ and $L_{T}$, we obtain the Kähler potential

$$
\begin{aligned}
K= & -\log \left(S+\bar{S}-A(T+\bar{T})+2 \delta \bar{M} e^{A} M-\epsilon\left|\alpha M^{3}\right|^{2}\right) \\
& -3 \log \left(T+\bar{T}-2 \bar{M} e^{A} M\right),
\end{aligned}
$$

and the gauge kinetic functions $f^{a}=c^{a} S+\left(\beta^{a}-A c^{a}\right) T$. This theory is clearly related to Eq. (4.13) by the holomorphic redefinition $S \rightarrow S-A T$, and the choice $A=\delta$ leads to theory (4.15).

This discussion shows that the counterterm $\Delta \mathcal{L}$ is irrelevant in the four-dimensional effective supergravity, that all values of $A$ lead to equivalent Lagrangians, with the same dynamical equations. Further information due, for instance, to compactification of extra dimensions could however appear more natural with a specific value of $A$, if one insists to use the version of the effective supergravity with a chiral dilaton multiplet. For instance, all corrections linear in $T$ appear as gauge thresholds with the choice $A=0$. But one could as well use the version with a linear dilaton which is free of ambiguities.

In addition, the holomorphic redefinition (4.14) mixes terms of different orders in the string loop expansion. As a consequence, the distinction between terms in $\mathcal{L}^{(0)}$ and corrections in $\mathcal{L}^{(1)}$ becomes ambiguous in general in the large $T$ limit.

The values of the coefficients $\delta$ and $\epsilon$ can be inferred from a direct calculation of the $M$-dependent anomaly-cancelling terms in M-theory on $O_{7}$. This is the subject of the next paragraph. Notice however that such a calculation only provides the terms of first order in the matter multiplets $\bar{M} e^{A} M$ and $\left|\alpha M^{3}\right|^{2}$. To this order, expression (4.13) becomes

$$
\begin{aligned}
K= & -\log (S+\bar{S})-3 \log \left(T+\bar{T}-2 \bar{M} e^{A} M\right) \\
& -\frac{1}{S+\bar{S}}\left[2 \delta \bar{M} e^{A} M-\epsilon\left|\alpha M^{3}\right|^{2}\right] .
\end{aligned}
$$

The term with coefficient $\delta$ has been obtained in direct Calabi-Yau reductions of Mtheory on $S^{1} / \mathbf{Z}_{2}$ (see for instance [6, 7] $\mathbb{E}^{\mathbb{2}}$ ). The charged matter contribution with coefficient $\epsilon$ was not included in these analyses.

The gauge contributions appearing in Eq. (4.10) read

$$
-2 \sum_{a}\left[\left\{c^{a}(S+\bar{S})+\beta^{a}\left(V_{T}+2 \bar{M} e^{A} M\right)\right\} \Omega^{a}\right]_{D},
$$

\footnotetext{
${ }^{17}$ It has also been obtained, in a quite different context, by Itoyama and Leon [26].
} 
so that the gauge coupling constants are given by

$$
\frac{1}{g_{a}^{2}}=c^{a} \operatorname{Re} s+\frac{1}{2} \beta^{a}\left(C_{T}+2 \bar{M} M\right) .
$$

This expression becomes harmonic once the Bianchi identity imposing $C_{T}+2 \bar{M} M=$ $2 \operatorname{Re} T$ has been used. Similarly, one obtains from Eq. (4.11)

$$
\frac{1}{g_{a}^{2}}=\frac{c^{a} \Upsilon}{4 C}+\beta^{a} \operatorname{Re} T-c^{a} \delta \bar{M} M+\frac{c^{a} \epsilon}{2}\left|\alpha M^{3}\right|^{2}
$$

with $\Upsilon=2\left(z_{0} \bar{z}_{0}\right)^{3 / 2}(\operatorname{Re} T-\bar{M} M)^{3 / 2} C^{-1 / 2}$. This second form of the gauge couplings is never harmonic since it is obtained from a theory with a linear dilaton multiplet. Both expressions do however agree since the chiral-linear duality relation between $\operatorname{Re} s$ and $C$ is

$$
\operatorname{Re} s=\frac{\Upsilon}{4 C}-\delta \bar{M} M+\frac{\epsilon}{2}\left|\alpha M^{3}\right|^{2}
$$

\subsection{On the eleven-dimensional origin of the anomaly-cancelling terms}

In ten dimensions, anomaly-cancelling terms for the $E_{8} \times E_{8}$ heterotic string are wellknown. There are two terms. The first couples a gauge and Lorentz invariant eight-form $\hat{X}_{8}$ to the two-form field $\hat{B}$. The second one is proportional to $\int\left(\Omega_{3,1}+\Omega_{3,2}-\Omega_{3 \mathrm{~L}}\right) \wedge \hat{X}_{7}$, with $d \hat{X}_{7}=\hat{X}_{8}$.

The anomaly-cancelling terms arising from M-theory on $S^{1} / \mathbf{Z}_{2}$ [1, 2] have recently been precisely computed [27]. They arise from the following action terms in eleven dimensions [1, 2], 28]:

$$
-\frac{\lambda^{2}}{(4 \pi)^{2} \kappa_{11}^{2}} \int G \wedge X_{7}-\frac{1}{12 \kappa_{11}^{2}} \int C \wedge G \wedge G,
$$

where

$$
X_{7}=\frac{1}{12(4 \pi)^{3}}\left(\frac{1}{2} \Omega_{7 \mathrm{~L}}-\frac{1}{8}\left(\operatorname{tr} R^{2}\right) \Omega_{3 \mathrm{~L}}\right),
$$

$\kappa_{11}$ is the eleven-dimensional gravitational constant and $\lambda$ is the gauge coupling constant on both ten-dimensional $\mathbf{Z}_{2}$-fixed planes, as defined by the gauge action (3.12). The Chern-Simons forms are defined by

$$
d \Omega_{7 \mathrm{~L}}=\operatorname{tr} R^{4}, \quad d \Omega_{3 \mathrm{~L}}=\operatorname{tr} R^{2}, \quad d \Omega_{3, i}=\operatorname{tr} F_{i}^{2}, \quad i=1,2 .
$$

Cancellation of gauge and gravitational anomalies and coherence of the reduction to ten dimensions impose $\lambda^{6}=(4 \pi)^{5} \kappa_{11}^{4} / 12$ [1, 2, 27]. This condition relates the gauge 
coupling $\lambda$ and the $S^{1}$ radius. Solving the Bianchi identity verified by the four-form field $G$ on $S^{1} / \mathbf{Z}_{2}$ and extracting the zero modes leads to the following Green-Schwarz terms

$$
\begin{aligned}
S_{G S}= & -\frac{1}{48 \pi} \int_{M_{10}} \hat{B} \wedge\left[\left(I_{4,1}\right)^{2}+\left(I_{4,2}\right)^{2}-I_{4,1} I_{4,2}+\frac{1}{(4 \pi)^{4}}\left(\frac{1}{2} \operatorname{tr} R^{4}-\frac{1}{8}\left(\operatorname{tr} R^{2}\right)^{2}\right)\right] \\
& -\frac{1}{(4 \pi)^{2}} \int_{M_{10}}\left(\Omega_{3,1}+\Omega_{3,2}-\Omega_{3 \mathrm{~L}}\right) \wedge X_{7} .
\end{aligned}
$$

In this expression,

$$
I_{4, i}=\frac{1}{(4 \pi)^{2}}\left(\operatorname{tr} F_{i}^{2}-\frac{1}{2} \operatorname{tr} R^{2}\right), \quad i=1,2
$$

and

$$
\hat{B}_{A B}=\frac{\lambda^{2}}{\kappa_{11}^{2}} \int_{S^{1}} d x^{4} C_{A B 4}
$$

As usual, the two-form field $\hat{B}$ couples to $\hat{X}_{8}$. But the second term has a particular structure: $\hat{X}_{7}$ is replaced by the purely gravitational seven-form (4.21): the anomalycancelling terms derived from M-theory differ from the standard expression of the heterotic string by a well-defined local counterterm, as permitted by the descent equations 27.

The Calabi-Yau compactification of $\hat{B}_{A B}$ leads to two zero modes of the bulk fields,

$$
\kappa^{2} \hat{B}_{\mu \nu}=\frac{\lambda^{2}}{V_{6}} C_{\mu \nu 4}, \quad \quad \kappa^{2} \hat{B}_{i \bar{j}}=\frac{\lambda^{2}}{V_{6}} C_{i \bar{j} 4},
$$

since $\frac{\lambda^{2}}{\kappa_{11}^{2}}=\frac{1}{\kappa^{2}} \frac{\lambda^{2}}{V_{6}} \frac{1}{V_{1}}$. By Eqs. (2.30), these states are related to our four-dimensional bulk multiplets $L($ or $S$ ) and $T$ by

$$
\hat{B}_{\mu \nu}=4 b_{\mu \nu}, \quad \quad \kappa^{2} \hat{B}_{i \bar{j}}=i \operatorname{Im} T \delta_{i \bar{j}} .
$$

The number $\lambda^{2} / V_{6}$ disappears in this identification: it does not play any role in the four-dimensional effective theory.

Our task is then to compute the reduction of $S_{G S}$ on $M_{4} \times($ Calabi-Yau). Since we restrict ourselves to contributions with at most two derivatives, we only need the reduction of

$$
\Delta=-\frac{1}{48 \pi} \int_{M_{10}} \hat{B} \wedge\left[\left(I_{4,1}\right)^{2}+\left(I_{4,2}\right)^{2}-I_{4,1} I_{4,2}\right] .
$$

\footnotetext{
${ }^{18}$ Recall that $x^{4}$ is the $S^{1}$ coordinate.
} 
The global definition of the four-form field $G$ ('cohomology condition') implies [4]

$$
\left\langle I_{4,1}\right\rangle=-\left\langle I_{4,2}\right\rangle
$$

for the Calabi-Yau background, which is a $(2,2)$ form. The counterterm $\Delta$ becomes then

$$
\begin{aligned}
\Delta= & -\frac{1}{4(4 \pi)^{3}} \int_{M_{10}} \hat{B} \wedge\left\langle I_{4,1}\right\rangle \wedge\left(\operatorname{tr} F_{1}^{2}-\operatorname{tr} F_{2}^{2}\right) \\
& -\frac{1}{12(4 \pi)^{5}} \int_{M_{10}} \hat{B} \wedge\left[\left(\operatorname{tr} F_{1}^{2}\right)^{2}+\left(\operatorname{tr} F_{2}^{2}\right)^{2}-\left(\operatorname{tr} F_{1}^{2}\right)\left(\operatorname{tr} F_{2}^{2}\right)\right]+\ldots,
\end{aligned}
$$

where gravitational contributions with more than two derivatives are omitted. Notice that the two $E_{8}$ factors contribute with opposite signs in the background-dependent term.

The standard embedding is defined by $\left\langle\operatorname{tr} F_{2}^{2}\right\rangle=0$, while $\left\langle\operatorname{tr} F_{1}^{2}\right\rangle$ is in the $S U(3)$ direction of the maximal embedding $E_{6} \times S U(3) \subset E_{8}$. As a consequence,

$$
\left\langle\operatorname{tr} F_{1}^{2}\right\rangle=\left\langle\operatorname{tr} R^{2}\right\rangle=2(4 \pi)^{2}\left\langle I_{4,1}\right\rangle,
$$

which in turn leads to

$$
\begin{aligned}
\Delta= & -\frac{1}{8(4 \pi)^{5}} \int_{M_{10}} \hat{B} \wedge\left\langle\operatorname{tr} R^{2}\right\rangle \wedge\left(\operatorname{tr} F_{1}^{2}-\operatorname{tr} F_{2}^{2}\right) \\
& -\frac{1}{12(4 \pi)^{5}} \int_{M_{10}} \hat{B} \wedge\left[\left(\operatorname{tr} F_{1}^{2}\right)^{2}+\left(\operatorname{tr} F_{2}^{2}\right)^{2}-\left(\operatorname{tr} F_{1}^{2}\right)\left(\operatorname{tr} F_{2}^{2}\right)\right]+\ldots
\end{aligned}
$$

To derive the zero modes of $\operatorname{tr} F_{1}^{2}$ and $\operatorname{tr} F_{2}^{2}$, it is simpler to consider the ChernSimons forms, using the relation

$$
\left(\operatorname{tr} F_{i}^{2}\right)_{A B C D}=4 \partial_{[A}\left(\Omega_{3, i}\right)_{B C D]}, \quad i=1,2 .
$$

In the standard embedding, the unbroken $E_{8}$ group generates a $N=1$ super-Yang-Mills multiplet only. The only massless mode is then

$$
\left(\operatorname{tr} F_{2}^{2}\right)_{A B C D} \longrightarrow\left(\operatorname{tr} F_{E_{8}}^{2}\right)_{\mu \nu \rho \sigma}, \quad\left(\Omega_{3,2}\right)_{A B C} \longrightarrow\left(\Omega_{E_{8}}\right)_{\mu \nu \rho} .
$$

Clearly, these massless modes of $\operatorname{tr} F_{2}^{2}$ and $\Omega_{3,2}$ are respectively components of the four-dimensional $N=1$ multiplets $\mathcal{W}^{2} \mathcal{W}^{2}$ and $\Omega^{2}$ used earlier. More precisely:

$$
\begin{aligned}
{\left[\mathcal{W}^{2} \mathcal{W}^{2}\right]_{\mathrm{f}-\text { component }} } & =-\frac{1}{2} \operatorname{tr} F_{E_{8} \mu \nu} F_{E_{8}}^{\mu \nu}-\frac{i}{4 \epsilon} \epsilon^{\mu \nu \rho \sigma} \operatorname{tr} F_{E_{8} \mu \nu} F_{E_{8} \rho \sigma}+\ldots, \\
{\left[\Omega^{2}\right]_{\mathrm{d}-\text { component }} } & =\frac{1}{16} \operatorname{tr} F_{E_{8} \mu \nu} F_{E_{8}}^{\mu \nu}+\ldots \\
{\left[\Omega^{2}\right]_{\text {vector component }} } & =\frac{1}{8 e} \epsilon^{\mu \nu \rho \sigma}\left(\Omega_{E_{8}}\right)_{\nu \rho \sigma}+\ldots
\end{aligned}
$$


The gauge fields of the $E_{8}$ group broken into $E_{6}$ generate $E_{6}$ gauge fields and the chiral matter multiplet $M$, transforming in representation 27. Accordingly, the massless modes of $\operatorname{tr} F_{1}^{2}$ are:

$$
\begin{aligned}
\left(\operatorname{tr} F_{1}^{2}\right)_{A B C D} & \longrightarrow \quad\left(\operatorname{tr} F_{E_{6}}^{2}\right)_{\mu \nu \rho \sigma}, \\
& \longrightarrow \quad\left(\operatorname{tr} F_{1}^{2}\right)_{\mu \nu i \bar{j}}=2 \partial_{[\mu}\left(\Omega_{3,1}\right)_{\nu] i \bar{j}} \\
& \longrightarrow \quad\left(\operatorname{tr} F_{1}^{2}\right)_{\mu i j k}=\partial_{\mu}\left(\Omega_{3,1}\right)_{i j k} \\
& \longrightarrow \quad\left(\operatorname{tr} F_{1}^{2}\right)_{\mu \overline{i j k}}=\partial_{\mu}\left(\Omega_{3,1}\right)_{\overline{i j k}}
\end{aligned}
$$

While as before $\left(\Omega_{3,1}\right)_{A B C} \rightarrow\left(\Omega_{E_{6}}\right)_{\mu \nu \rho}$, the other components of $\Omega_{3,1}$ involve the scalar component of the matter multiplet $M$ and require more care since we have already precisely defined the four-dimensional field $M$ by its coupling to the bulk fields. To obtain the correct relations, a detour is helpful.

As already explained, the gauge kinetic action (3.12) also generates the four-dimensional kinetic terms for the matter multiplet $M$. In the four-dimensional effective Lagrangian, these contributions arise as the highest component of the 'matter ChernSimons multiplet' $\bar{M} e^{A} M$, which includes $-2\left(D_{\mu} \bar{M}\right)\left(D^{\mu} M\right)$. This multiplet also contains in its vector component the matter Chern-Simons form

$$
\Omega_{\mu}^{M}=i \bar{M}\left(D_{\mu} M\right)-i\left(D_{\mu} \bar{M}\right) M
$$

This is completely similar to the gauge Chern-Simons multiplet which includes gauge kinetic terms in its d-component and $\left(\Omega_{3}\right)_{\mu \nu \rho}$ in its vector component, as indicated by expressions (4.27). A direct computation of the relation between kinetic terms due to the action (3.12), and the highest component of $\bar{M} e^{A} M$ delivers then the relation between $\left(\Omega_{3,1}\right)_{\mu i \bar{j}}$ and this multiplet, by four-dimensional supersymmetry. A similar operation gives the relation between $\left(\Omega_{3,1}\right)_{i j k}$ and the superpotential multiplet $\alpha M^{3}$. The relations are

$$
\begin{aligned}
\left(\Omega_{3,1}\right)_{\mu i \bar{j}} & =\frac{1}{6 \kappa^{2}}\left[\left(D_{\mu} \bar{M}\right) M-\bar{M}\left(D_{\mu} M\right)\right] \delta_{i \bar{j}}=\frac{i}{6 \kappa^{2}} \delta_{i \bar{j}}\left[\bar{M} e^{A} M\right]_{\text {vector component }}, \\
\left(\Omega_{3,1}\right)_{i j k} & =\frac{1}{3 \kappa^{3}} \alpha \bar{M}^{3} \epsilon_{i j k}=\frac{1}{3 \kappa^{3}} \epsilon_{i j k}\left[\alpha \bar{M}^{3}\right]_{\text {scalar component }}
\end{aligned}
$$

together with the last equation (4.27) which applies to all gauge Chern-Simons forms.

With the complete identification of the massless modes of the Chern-Simons threeforms, we are equipped for translating the Calabi-Yau reduction of the Green-Schwarz 
counterterm (4.26) in a four-dimensional supergravity density formula. After straightforward manipulations of Eq. (4.26), we obtain

$$
\begin{aligned}
\mathcal{L}_{G S}= & -\frac{1}{192(4 \pi)^{5}} I \epsilon^{\mu \nu \rho \sigma} \frac{\lambda^{2}}{V_{6}}\left(\partial_{\mu} a\right)\left(\Omega_{E_{6}}-\Omega_{E_{8}}\right)_{\nu \rho \sigma} \\
& +\frac{i}{384(4 \pi)^{5} \kappa^{2}} I \epsilon^{\mu \nu \rho \sigma} \frac{\lambda^{2}}{V_{6}}\left(\partial_{\mu} C_{\nu \rho 4}\right)\left(M D_{\sigma} \bar{M}-\bar{M} D_{\sigma} M\right) \\
& +\frac{i \alpha^{2}}{216(4 \pi)^{5}} \frac{V_{6}}{\kappa^{6}} \epsilon^{\mu \nu \rho \sigma} \frac{\lambda^{2}}{\kappa^{2} V_{6}}\left(\partial_{\mu} C_{\nu \rho 4}\right)\left[\bar{M}^{3}\left(\partial_{\sigma} M^{3}\right)-\left(\partial_{\sigma} \bar{M}^{3}\right) M^{3}\right]+\ldots \\
= & \frac{1}{384(4 \pi)^{5}} I \epsilon^{\mu \nu \rho \sigma} T_{\mu}\left(\Omega_{E_{6}}-\Omega_{E_{8}}\right)_{\nu \rho \sigma} \\
& -\frac{i}{96(4 \pi)^{5}} I \epsilon^{\mu \nu \rho \sigma}\left(\partial_{\nu} b_{\rho \sigma}\right)\left(M D_{\mu} \bar{M}-\bar{M} D_{\mu} M\right) \\
& -\frac{i \alpha^{2}}{54(4 \pi)^{5}} \frac{V_{6}}{\kappa^{6}} \epsilon^{\mu \nu \rho \sigma}\left(\partial_{\nu} b_{\rho \sigma}\right)\left[\bar{M}^{3}\left(\partial_{\mu} M^{3}\right)-\left(\partial_{\mu} \bar{M}^{3}\right) M^{3}\right]+\ldots
\end{aligned}
$$

where the dots indicate the terms required by $N=1$ supersymmetry and $I$ is the dimensionless integral

$$
I=\kappa^{-2} \int_{C Y} d V_{6} \delta_{i \bar{i}} \epsilon^{i j k} \epsilon^{\overline{i j k}}\left\langle\operatorname{tr} R^{2}\right\rangle_{j k \overline{j k}}
$$

in terms of the $(1,1)$ (the metric tensor), $(3,0)$ and $(0,3)$ Calabi-Yau tensors and the background $\left\langle\operatorname{tr} R^{2}\right\rangle$.

Using Eqs. (4.28), we can write the Green-Schwarz counterterm in superfield form as:

$$
\begin{aligned}
\mathcal{L}_{G S}= & -\frac{I}{48(4 \pi)^{5}}\left[\left(V_{T}+2 \bar{M} e^{A} M\right)\left(\Omega^{1}-\Omega^{2}\right)\right]_{D}-\frac{I}{48(4 \pi)^{5}}\left[V \bar{M} e^{A} M\right]_{D} \\
& +\frac{1}{27(4 \pi)^{5}} \frac{V_{6}}{\kappa^{6}}\left[V\left|\alpha M^{3}\right|^{2}\right]_{D} .
\end{aligned}
$$

Comparing the three terms of $\mathcal{L}_{G S}$ with the corresponding parts of (4.10), we can express the coefficients $\beta^{a}, \delta$ and $\epsilon$ in terms of the Calabi-Yau dependent integral $I$ :

$$
\delta=\beta^{1}=-\beta^{2}=\frac{I}{96(4 \pi)^{5}} \quad, \quad \epsilon=\frac{1}{27(4 \pi)^{5}} \frac{V_{6}}{\kappa^{6}} .
$$

The calculation predicts then $\beta^{1}=-\beta^{2}=\delta$, a result already obtained in references [6, 7], for instance. Once again, we stress that we have obtained next-order corrections to the effective Wilson Lagrangian. As argued earlier in paragraph 4.1, while we expect $\beta^{1}$ and $\beta^{2}$ to have physical significance as coefficients of the modulus-dependent threshold corrections, the parameter $\delta$ is not necessarily a physical quantity. To decide of its relevance, a calculation at the same order of threshold corrections in the effective action should be performed, but this computation requires a detailed knowledge of the charged matter spectrum and couplings. 


\section{Conclusions}

In this paper, we have deduced the structure of the four-dimensional $N=1$ effective (wilsonnian) supergravity describing the universal massless sector of M-theory compactified on $(\mathrm{CY})_{3} \times S^{1} / \mathbf{Z}_{2}$. The theory depends on three categories of multiplets: 'M-theory multiplets' $V, V_{T}$ and $W$ describe the degrees of freedom of the M-theory four-index tensor, 'source multiplets' $\Omega^{a}, \bar{M} e^{A} M$ and $\alpha M^{3}$ are related to the source terms in M-theory Bianchi identities and 'Lagrange multiplets' $S, L_{T}$ and $U$ impose by their field equations the Bianchi identities. In addition, the multipliers $S$ and $L_{T}$ generate the four-dimensional axion-tensor duality which is known to be an important ingredient of the formulation of the string dilaton beyond the lowest order.

An effective supergravity similar to expression (4.10) is in principle valid for generic compactifications of M-theory with unbroken $N=1$ supersymmetry in four dimensions. One needs to identify the appropriate supermultiplets appearing as sources in the Bianchi identities generated by $S, L_{T}$ and $U$. Only the values of coefficients like $\beta^{a}, \delta$ and $\epsilon$ depend on the detailed geometry of the compact space. This method is especially useful in deriving contributions to the effective Lagrangian due to non-perturbative states like M-theory five-brane degrees of freedom or condensates. Some of these more general $N=1$ vacua will be studied in a forthcoming publication [9].

\section{Acknowledgements}

The authors have benefited from discussions with A. Bilal, C. Kounnas, A. Lukas, D. Lüst and B. Ovrut. This research was supported in part by the European Union under the TMR contract ERBFMRX-CT96-0045, the Swiss National Science Foundation and the Swiss Office for Education and Science.

\section{Appendix: Notations and conventions}

\section{Metrics and coordinates:}

The space-time metric has signature $(-,+,+, \ldots,+)$.

For coordinates, our notation is: 
$D=11$ curved space-time:

$D=10$ curved space-time:

$D=4$ curved space-time:

$S_{1} / \mathbf{Z}_{2}$ direction:

Calabi-Yau directions:

Calabi-Yau complex (Käl

$$
\begin{array}{ll}
x^{M} & M=0, \ldots, 10 \\
x^{A} & \\
x^{\mu} & \mu=0,1,2,3 \\
x^{4} & \\
x^{a} & a=5, \ldots, 10
\end{array}
$$$$
z^{i}, \bar{z}^{\bar{i}} \quad i=1,2,3
$$

For reduction purposes, we simply use

$$
z^{l}=\frac{1}{\sqrt{2}}\left(x^{l}+i x^{l+3}\right), \quad \quad \bar{z}^{\bar{l}}=\frac{1}{\sqrt{2}}\left(x^{l}-i x^{l+3}\right), \quad l=1,2,3 .
$$

$\epsilon_{i j k}$ is the $S U(3)$-invariant Calabi-Yau tensor such that $\epsilon_{123}=\epsilon_{\overline{123}}=1$.

Antisymmetric tensors:

Antisymmetrization of $n$ indices has unit weight:

$$
A_{\left[M_{1} \ldots M_{n}\right]}=\frac{1}{n !}\left(A_{M_{1} \ldots M_{n}} \pm(n !-1) \text { permutations }\right) .
$$

\section{Differential forms}

For a $p$-index antisymmetric tensor, we define

$$
A^{(p)}=\frac{1}{p !} A_{M_{1} \ldots M_{p}} d x^{M_{1}} \wedge \ldots \wedge d x^{M_{p}} .
$$

Then,

$$
\begin{aligned}
& A^{(p)} \wedge B^{(q)}=\frac{1}{p ! q !} A_{M_{1} \ldots M_{p}} B_{M_{p+1} \ldots M_{p+q}} d x^{M_{1}} \wedge \ldots \wedge d x^{M_{p+q}}=C^{(p+q)}, \\
& C_{M_{1} \ldots M_{p+q}}=\frac{(p+q) !}{p ! q !} A_{\left[M_{1} \ldots M_{p}\right.} B_{\left.M_{p+1} \ldots M_{p+q}\right]} .
\end{aligned}
$$

The exterior derivative is $d=\partial_{M} d x^{M}$. The curl $F^{(p+1)}=d A^{(p)}$ of a $p$-form reads then

$$
\begin{aligned}
d A^{(p)} & =\frac{1}{p !}\left(\partial_{M} A_{N_{1} \ldots N_{p}}\right) d x^{M} \wedge d x^{N_{1}} \wedge \ldots \wedge d x^{N_{p}} \\
& =\frac{1}{(p+1) !} F_{M_{1} \ldots M_{p+1}} d x^{M_{1}} \wedge \ldots \wedge d x^{M_{p+1}}, \\
F_{M_{1} \ldots M_{p+1}} & =(p+1) \partial_{\left[M_{1}\right.} A_{\left.M_{2} \ldots M_{p+1}\right]} \\
& =\partial_{M_{1}} A_{M_{2} \ldots M_{p+1}} \pm p \text { cyclic permutations }
\end{aligned}
$$

The volume form in $D$ space-time dimensions is $d x^{M_{1}} \wedge \ldots \wedge d x^{M_{D}}=\epsilon^{M_{1} \ldots M_{D}} d^{D} x$.

We use analogous conventions for forms in four space-time dimensions.

\section{References}

[1] P. Hořava and E. Witten, Nucl. Phys. B460 (1996) 506, (hep-th/9510209). 
[2] P. Hořava and E. Witten, Nucl. Phys. B475 (1996) 94, (hep-th/9603142).

[3] E. Cremmer, B. Julia and J. Scherk, Phys. Lett. 76B (1978) 409.

[4] E. Witten, Nucl. Phys. B471 (1996) 135, (hep-th/9602070).

[5] T. Banks and M. Dine, Nucl. Phys. B479 (1996) 173, hep-th/9605136);

E. Caceres, V. S. Kaplunovsky and I. M. Mandelberg, Nucl. Phys. B493 (1997) 73, (hep-th/9606036);

P. Hořava, Phys. Rev. D54 (1996) 7561, (hep-th/9608019);

I. Antoniadis and M. Quirós, Phys. Lett. B392 (1997) 61, (hep-th/9609209); Nucl. Phys. B505 (1997) 109, (hep-th/9705037); Phys. Lett. B416 (1998) 327, hepth/9707208);

H.-P. Nilles and S. Stieberger, Nucl. Phys. B499 (1997) 3, (hep-th/9702110);

E. Dudas and C. Grojean, Nucl. Phys. B507 (1997) 553, (hep-th/9704177);

Z. Lalak and S. Thomas, Nucl. Phys. B515 (1998) 55, (hep-th/9707223);

A. Lukas, B. A. Ovrut and D. Waldram, Phys. Rev. D57 (1998) 7529, hhepth/9711197);

E. A. Mirabelli and M. E. Peskin, Phys. Rev. D58 (1998) 065002, hepth/9712214);

J. Ellis, Z. Lalak, S. Pokorski and W. Pokorski, Nucl. Phys. B540 (1999) 149, (hep-ph/9805377);

J. Ellis, Z. Lalak, S. Pokorski and S. Thomas, (hep-th/9906148).

[6] H.-P. Nilles, M. Olechowski and M. Yamaguchi, Phys. Lett. B415 (1997) 24, hepth/9707143); Nucl. Phys. B530 (1998) 43, (hep-th/9801030).

[7] A. Lukas, B. A. Ovrut, D. Waldram, Nucl. Phys. B532 (1998) 43, thepth/9710208).

[8] Z. Lalak, S. Pokorski and S. Thomas, Nucl. Phys. B549 (1999) 63, (hep-ph/9807503);

A. Lukas, B. A. Ovrut and D. Waldram, Phys. Rev. D59 (1999) 106005, hepth/9808101.

[9] J.-P. Derendinger and R. Sauser, in preparation. 
[10] S. Ferrara, L. Girardello, T. Kugo and A. Van Proeyen, Nucl. Phys. B223 (1983) 191.

[11] T. Kugo and S. Uehara, Nucl. Phys. B226 (1983) 49; see also: Nucl. Phys. B222 (1983) 125.

[12] E. Cremmer, S. Ferrara, L. Girardello and A. Van Proeyen, Phys. Lett. B116 (1982) 231.

[13] J.-P. Derendinger, S. Ferrara, C. Kounnas and F. Zwirner, Nucl. Phys. B372 (1992) 145.

[14] S. Cecotti, S. Ferrara and M. Villasante, Int. J. Mod. Phys. A2 (1987) 1839.

[15] J.-P. Derendinger, F. Quevedo and M. Quirós, Nucl. Phys. B428 (1994) 282, hepth/9402007).

[16] E. Witten, Phys. Lett. $\underline{\text { B155 }}$ (1985) 151.

[17] L. Dixon, V. Kaplunovsky and J. Louis, Nucl. Phys. B355 (1991) 649.

[18] J.-P. Derendinger, L. E. Ibáñez and H.-P. Nilles, Phys. Lett. B155 (1985) 65.

[19] J.-P. Derendinger, L. E. Ibáñez and H.-P. Nilles, Nucl. Phys. B267 (1986) 365.

[20] C. P. Burgess, A. Font and F. Quevedo, Nucl. Phys. B272 (1986) 661.

[21] L. Dixon, V. Kaplunovsky and J. Louis, Nucl. Phys. B329 (1990) 27.

[22] G. F. Chapline and N. S. Manton, Phys. Lett. B120 (1983) 105.

[23] M. B. Green and J. H. Schwarz, Phys. Lett. B149 (1984) 117.

[24] J. Louis, in: 'Particles, Strings and Cosmology', ed. by P. Nath and S. Reucroft (World Scientific, Singapore, 1992), p. 751-765.

[25] G. L. Cardoso and B. A. Ovrut, Nucl. Phys. B369 (1992) 351; B392 (1993) 315, (hep-th/9205009).

[26] H. Itoyama and J. Leon, Phys. Rev. Lett. $\underline{56}$ (1986) 2352. 
[27] A. Bilal, J.-P. Derendinger and R. Sauser, (hep-th/9912150), to appear in Nucl. Phys. B.

[28] C. Vafa and E. Witten, Nucl. Phys. B447 (1995) 261, (hep-th/9505053);

M. J. Duff, J. T. Liu and R. Minasian, Nucl. Phys. B452 (1995) 261, thepth/9506126);

S. P. de Alwis, Phys. Lett. B392 (1997) 332, (hep-th/9609211). 